
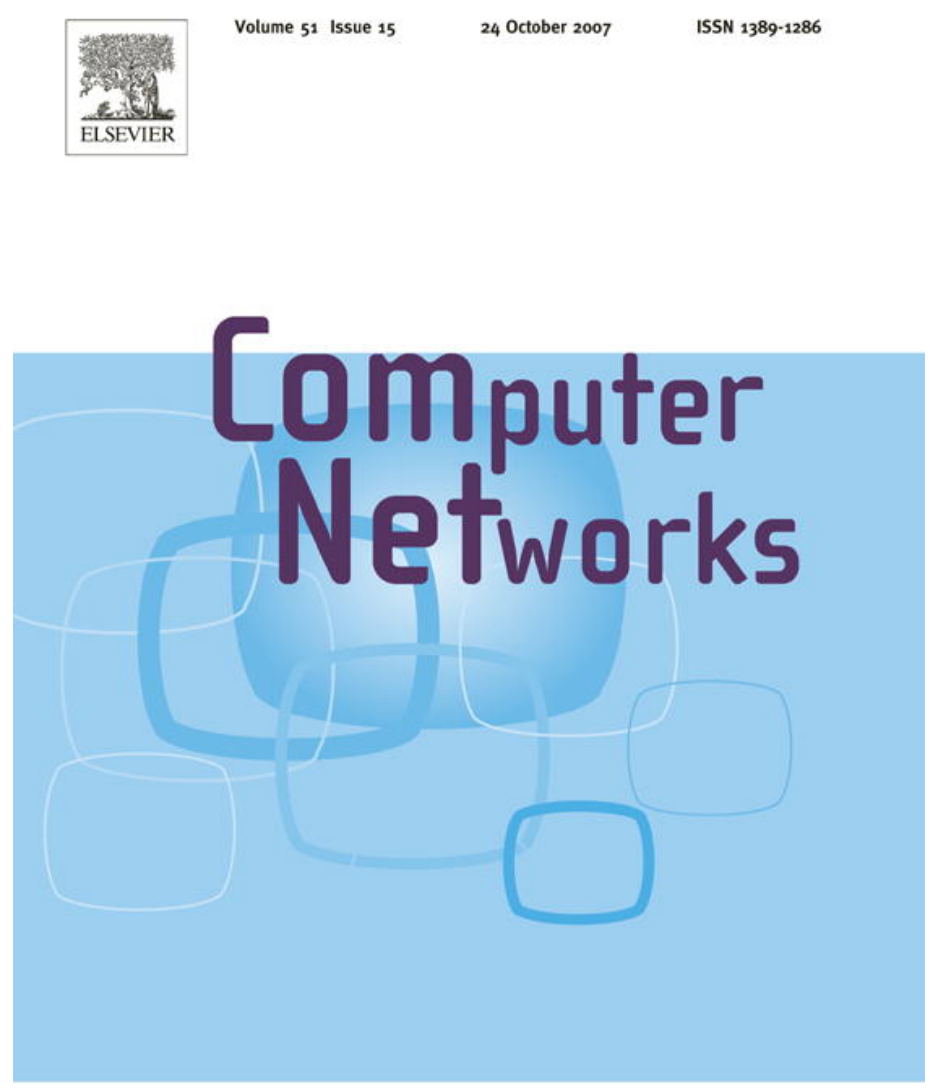

This article was published in an Elsevier journal. The attached copy

is furnished to the author for non-commercial research and education use, including for instruction at the author's institution, sharing with colleagues and providing to institution administration.

Other uses, including reproduction and distribution, or selling or licensing copies, or posting to personal, institutional or third party websites are prohibited.

In most cases authors are permitted to post their version of the article (e.g. in Word or Tex form) to their personal website or institutional repository. Authors requiring further information regarding Elsevier's archiving and manuscript policies are encouraged to visit: 


\title{
Stability and TCP-friendliness of AIMD/RED systems with feedback delays
}

\author{
Lijun Wang ${ }^{\text {a }}$, Lin Cai ${ }^{\mathrm{b}}$, Xinzhi Liu ${ }^{\mathrm{a}}$, Xuemin (Sherman) Shen ${ }^{\mathrm{c}, *}$ \\ a Department of Applied Mathematics, University of Waterloo, Waterloo ON, Canada N2L $3 G 1$ \\ ${ }^{\mathrm{b}}$ Department of Electrical and Computer Engineering, University of Victoria, Victoria, BC, Canada V8W $3 P 6$ \\ ${ }^{\mathrm{c}}$ Department of Electrical and Computer Engineering, University of Waterloo, Waterloo ON, Canada N2L $3 G 1$
}

Received 25 March 2007; received in revised form 22 May 2007; accepted 25 June 2007

Available online 6 July 2007

Responsible Editor: L.G. Xue

\begin{abstract}
In this paper, we systematically study the stability of a class of generalized Additive Increase and Multiplicative Decrease/Random Early Detection (AIMD/RED) system. Sufficient conditions are obtained for asymptotic stability of both homogeneous-flow system and heterogeneous-flow system with or without feedback delay by using indirect Lyapunov and Lyapunov-Razumikhin method. Our study reveals the relationship between the AIMD parameters and the average window size of competing AIMD flows. Consequently, the Transmission Control Protocol (TCP)-friendly condition is derived. Numerical results with Matlab and simulation results with NS-2 are given to validate the theorems and analytical results. The analysis and the stability conditions derived can be used as a guideline to set up the AIMD/RED system parameters in order to maintain network stability and integrity, and to enhance system performance.
\end{abstract}

(c) 2007 Elsevier B.V. All rights reserved.

Keywords: Network stability; TCP-friendliness; Delay; AIMD; RED

\section{Introduction}

The proliferation and universal adoption of the Internet have escalated it as the key information transport platform. As a decentralized system, network stability and integrity rely on the end-to-end congestion control algorithm, which is deployed in

\footnotetext{
* Corresponding author. Tel.: +1 519888 4567x2691; fax: +1 5197463077.

E-mail address: xshen@bbcr.uwaterloo.ca (Xuemin (Sherman) Shen).
}

the dominant transport layer protocol, Transmission Control Protocol (TCP). A TCP sender interprets packet losses as congestion indicators and throttles the traffic load once a packet loss is detected, using an Additive Increase and Multiplicative Decrease (AIMD) [1] congestion control mechanism, aiming to avoid network congestion collapse. Specifically, a sender additively increases the sending rate to probe for available bandwidth when no congestion occurs and exponentially (multiplicatively) decreases its sending rate in response to network congestion. The additive increase rate 
of TCP is one packet per round-trip time ( $\mathrm{rtt}$ ) and the multiplicative decrease ratio of TCP is $1 / 2$. To support heterogeneous traffic, the general AIMD congestion control uses a pair of parameters $(\alpha, \beta)$ to set the increase rate and the decrease ratio [46]. On the other hand, to distribute the network congestion indicators fairly to all on-going flows, active queue management (AQM) [8,22], e.g., the Random Early Detection (RED) queue management scheme, has been proposed to be deployed in the intermediate nodes. With the RED schemes $[10,11]$, the intermediate nodes discard packets of all on-going flows randomly when the queue length exceeds a pre-defined threshold; therefore, the packet loss rate of each flow is roughly proportional to the flow sending rate. The AIMD congestion control, coupled with the RED queue in the core network, has been acknowledged as one of the key factors to the overwhelming success of the Internet.

With the rapid advances in optical and wireless communications, the Internet is becoming a more heterogeneous and diverse system: link capacity varies from several Kbps to several Gbps, with six orders of magnitude; transmission bit error rates vary from $<10^{-9}$ to $10^{-3}$, also with about six orders of magnitude; and end-to-end delay varies from several milliseconds to several seconds. A critical and immediate question is whether the AIMD/RED system is a stable, fair, and efficient system, independent of the heterogeneity of the link capacity, end-to-end delay, and network topology. In other words, should we re-design the Internet congestion control mechanism to accommodate future killer applications over the ever-diversified Internet, or can we take an incremental approach of engineering the existing congestion control mechanism and routers' queue management parameters to achieve the same objective?

Stability problems of TCP flows with RED queues have been extensively investigated in [1722]. New control mechanisms based on control theory and game theory have also been proposed [2]. Instead of proposing a new control mechanism, we focus on the stability and performance of the dominant AIMD congestion control mechanism with RED queues. In [22], using a fluid model, the global asymptotic stability of TCP/RED is proved, neglecting the feedback delay. The dynamics of TCP/RED with feedback delay has been studied using a frequency domain approach in [3]. Because of the heterogeneity of the Internet, understanding the stability conditions of the general AIMD/RED sys- tem with heterogeneous flows and feedback delays is critical for future network planning and design.

In this paper, we systematically study the stability of the AIMD/RED system, considering heterogeneous flows with different AIMD parameters in both delay-free marking and delayed marking scenarios. The definitions of stability and asymptotic stability follow that in [24]. Consider dynamic systems with time delay of the following form:

$\frac{\mathrm{d} x}{\mathrm{~d} t}=f\left(t, x(t), x\left(t-\tau_{1}(t)\right), \ldots, x\left(t-\tau_{m}(t)\right)\right)$,

where $x \in R^{n}, f: I \times R^{n} \times R^{n} \times \cdots \times R^{n} \rightarrow R^{n}$ is continuous. Let $\tau=\sup _{i=1, \ldots, m} \tau_{i}(t)$.

The trivial solution of the system is said to be

stable if for every $\epsilon>0$ and $t_{0} \in \mathbb{R}_{+}$, there exists some $\delta=\delta\left(t_{0}, \epsilon\right)>0$ such that for any $\xi(t) \in$ $C\left[\left[t_{0}-\tau, t_{0}\right], R^{n}\right],\|\xi\|<\delta$ implies $\left\|x\left(t, t_{0}, \xi\right)\right\|<\epsilon$ for all $t \geqslant t_{0}$;

asymptotically stable if the system is stable and for every $t_{0} \in \mathbb{R}_{+}$, there exists some $\eta=\eta\left(t_{0}\right)>0$ such that $\lim _{t \rightarrow \infty}\left\|x\left(t, t_{0}, \xi\right)\right\|=0$ whenever $\|\xi\|<\eta$.

Based on the fluid model of the generalized AIMD/RED system, we apply the methods of Lyapunov functional and Lyapunov function with Lyapunov-Razumikhin condition to study the stability properties of the system. Different sufficient conditions are derived for the local asymptotic stability of the system with feedback delays. Since the fluid model captures the ensemble averages of the system parameters, with the sufficient conditions derived, the ensemble averages or the time averages (over a round) of the AIMD/RED system can be locally asymptotically stable, even with heterogeneous feedback and propagation delays, so the AIMD/RED system can be marginally stable. A round is defined as the time interval between two instants at which the sender reduces its window size consecutively. The analysis also reveals the relationship between AIMD parameters and the average window size of competing AIMD flows, and the TCP-friendly condition is also derived. Numerical results are given to validate the analysis. Extensive simulations with NS-2 [7] are performed to study the system performance with realistic protocols and network topologies. The analytical and simulation results can help to better understand the stability and performance of AIMD/RED system, and the theoretical results can be used as a guideline 
for the setting of system parameters to maintain network stability and enhance system performance.

The remainder of the paper is organized as follows. Section 2 briefly introduces the related work. Section 3 proposes the model of the generalized AIMD/RED system. Section 4 studies the stability property of the generalized AIMD/RED system with delay-free marking, and derives the TCPfriendly condition and average queuing delay. The stability and fairness analysis of AIMD/RED system with heterogeneous feedback delays are given in Section 5. Numerical results with MATLAB and simulation results with NS-2 are presented in Section 6 , followed by concluding remarks and further research issues in Section 7.

\section{Related work}

Congestion control mechanisms and AQM schemes for the Internet have been extensively studied, aiming to achieve quick convergence to efficiency, stability, fair bandwidth sharing, and low packet loss rate.

Internet stability properties and fairness issues in the presence of feedback delay have received much attention recently. The original work of proposing the congestion controller using utility optimization has been done in [12]. Since then, lots of work have been conducted for the TCP/Random Exponential Marking (REM) system. For example, for the case of a single node and a single source in the TCP/ REM system, the design of congestion controllers and the stability problems with delays are studied in $[2,13,14]$, and the sufficient conditions for global stability are given as well. Recently, a discrete congestion control system has been proposed in [15] to maintain both stability and fairness under heterogeneous delayed feedback. The boundedness and stability for the TCP/REM system are discussed in [16].

In the design of congestion controllers, one of the important criteria is asymptotic stability, i.e., the capability of the network to avoid oscillations in the steady state and to properly respond to other external perturbations. AQM schemes recently discussed include RED, REM, Proportional-Integral (PI) control and Loss Ratio-based RED (LRED). For TCP/RED system, the sufficient conditions for global stability in the absence of feedback delay are given in [22]; the conditions for the stability of $\mathrm{TCP} / \mathrm{RED}$ system in the frequency domain are given in [3] by Nyquist stability criterion. The design and analysis of the PI controller for RED routers are discussed in [19]. Newly proposed AQM scheme, LRED in [9], measures the latest packet loss ratio, and uses it as a complement to queue length for adaptively adjusting the packet drop probability. To the best of our knowledge, the stability properties of AIMD/RED systems in the presence of heterogeneous AIMD and TCP flows with heterogeneous feedback delays have not been studied, and they are the main focus of this paper.

\section{A fluid-flow model of generalized AIMD/RED system}

A stochastic model of TCP behavior was developed using fluid-flow and stochastic differential equation analysis [18]. Simulation results have demonstrated that this model accurately captures the dynamics of TCP. We extend the fluid-flow model for general $\operatorname{AIMD}(\alpha, \beta)$ congestion control: the window size is increased by $\alpha$ packet per $r t$ if no packet loss occurs; otherwise, it is reduced to $\beta$ times its current value.

We first consider the case that all AIMD-controlled flows have the same $(\alpha, \beta)$ parameter pair and round-trip delay. The AIMD/RED fluid model relates to the ensemble averages of key network variables, and it is described by the following coupled, nonlinear differential equations:

$$
\begin{aligned}
& \frac{\mathrm{d} W(t)}{\mathrm{d} t}=\frac{\alpha}{R(t)}-\frac{2(1-\beta)}{1+\beta} W(t) \frac{W(t-R(t))}{R(t-R(t))} p(t-R(t)), \\
& \frac{\mathrm{d} q(t)}{\mathrm{d} t}= \begin{cases}\frac{N(t) \cdot W(t)}{R(t)}-C, & q>0, \\
\left\{\frac{N(t) \cdot W(t)}{R(t)}-C\right\}^{+}, & q=0,\end{cases}
\end{aligned}
$$

where $\{a\}^{+}=\max \{a, 0\}, \alpha>0, \quad \beta \in[0,1], \quad W \in[1$, $\left.W_{\max }\right]$ is the ensemble average of AIMD window size (packets); $q \in\left[0, q_{\mathrm{max}}\right]$ is the ensemble average of queue length (packets); $R$ is the round-trip time with $R(t)=\frac{q(t)}{C}+T_{p}(\mathrm{~s})$, where $C$ is the queue capacity (packets/s) and $T_{p}$ is the deterministic delay (including propagation, processing, and transmission delay). $N$ is the number of AIMD flows and $p$ is the probability of a packet being marked (or dropped).

The first differential equation of system (1) describes the $\operatorname{AIMD}(\alpha, \beta)$ window control dynamic. Roughly speaking, $\alpha / R$ represents the window's additive increase, while $\frac{2(1-\beta)}{1+\beta} W$ represents the window's multiplicative decrease in response to packet marking (or dropping) probability $p$. This is because the flow's 
window size always oscillates between $\beta W_{\max }$ to $W_{\max }$, the average window size over a round is $(1+\beta) W_{\max } / 2$. Each time, the window size is cut by $(1-\beta) W_{\max }=2(1-\beta) W /(1+\beta)$. The second equation models the bottleneck queue length as simply an accumulated difference between packet arrival rate $N W / R$ and link capacity $C .\{\cdot\}^{+}$in the model guarantees queue length is a non-negative number.

With RED, the packet marking probability is proportional to the average queue length: $p=K_{p}\left(q_{\text {act }}-\min _{\text {th }}\right) \quad$ with $\quad K_{p}>0$ and $p \in[0,1]$. When the actual queue length is less than or equal to the minimum threshold, i.e. $q_{\text {act }} \leqslant \min _{\text {th }}$, the marking probability is zero. Therefore, $\frac{\mathrm{d} W(t)}{\mathrm{d} t}=\frac{\alpha}{R}$, that is, the window size will keep increasing and not converge. In the following, we will discuss the stability property of this model when $q_{\text {act }}>\min _{\text {th }}$. Without loss of generality, let $q(t)=q_{\text {act }}(t)-\min _{\text {th }}$. Since the system behaves the same as a Drop-Tail queue once the queue length exceeds the maximum threshold $\max _{\mathrm{th}}$, to focus on the behavior of AIMD/RED, we choose $\max _{\text {th }}$ to be sufficiently large such that $p_{\max }=1$.

It should be noted that, (1) is a generalized AIMD/RED congestion control model, which includes the model studied in [18-23]. If we choose $\alpha=1, \beta=0.5$, (1) is equivalent to the traditional TCP/RED model. We will also show in the next section that the stability properties of the specific model in the literature is compatible with the corresponding properties of this generalized model as well.

\section{Stability and fairness analysis with delay-free marking}

\subsection{Stability of homogeneous AIMD/RED system}

With the fluid-flow model (1), we assume that the traffic load ( $N$ AIMD flows) is time-invariant, i.e., $N(t)=N$, and the round-trip time of each flow is a constant, $R(t)=R$. In the case of delay-free marking, i.e., $p=K_{p} q(t)$, the original delay-free marking model (1) can be written as a closed-loop dynamics:

$$
\begin{aligned}
& \frac{\mathrm{d} W(t)}{\mathrm{d} t}=\frac{\alpha}{R}-\frac{2(1-\beta)}{1+\beta} W(t) \frac{W(t)}{R(t)} K_{p} q(t), \\
& \frac{\mathrm{d} q(t)}{\mathrm{d} t}= \begin{cases}\frac{N \cdot W(t)}{R}-C, & q>0, \\
\left\{\frac{N \cdot W(t)}{R}-C\right\}^{+}, & q=0 .\end{cases}
\end{aligned}
$$

For a single-bottleneck system, the equilibrium point $\left(W_{0}^{*}, q_{0}^{*}\right)$ for (2) is given by
$W_{0}^{*}=\frac{R C}{N} ; \quad q_{0}^{*}=\frac{\alpha(1+\beta) N^{2}}{2(1-\beta) R^{2} C^{2} K_{p}}$.

At equilibrium, the RED queue length is inversely proportional to $K_{p}$. Thus, we should choose $K_{p}$ according to the delay budget.

With the transformed variables $\widetilde{W}:=W-$ $W_{0}^{*}, \tilde{q}:=q-q_{0}^{*}$, (2) becomes

$$
\begin{aligned}
\dot{\tilde{W}}(t)= & -\frac{2(1-\beta)}{1+\beta} \frac{\left(\widetilde{W}(t)+W_{0}^{*}\right)^{2}}{R} K_{p} \tilde{q}(t) \\
& -\frac{2(1-\beta)}{1+\beta} \frac{\widetilde{W}^{2}(t)+2 \widetilde{W}(t) W_{0}^{*}}{R} K_{p} q_{0}^{*}, \\
\dot{\tilde{q}}(t)= & \frac{N}{R} \cdot \widetilde{W}(t) .
\end{aligned}
$$

The equilibrium point of (4) is $\left(\widetilde{W}^{*}, \tilde{q}^{*}\right)=(0,0)$.

We construct the positive-definite Lyapunov function,

$V(\widetilde{W}, \tilde{q})=\frac{(1+\beta) N^{3}}{2(1-\beta) R^{2} C^{2}} \cdot \widetilde{W}^{2}(t)+\frac{1}{2} K_{p} \tilde{q}^{2}(t)$,

which is used to derive the following theorem.

Theorem 1. The equilibrium point of (2) is asymptotically stable for all $K_{p}>0$.

The proof of Theorem 1 is omitted, and we will prove a more general theorem (Theorem 2) in the next subsection.

From the viewpoint of control theory, the block diagram of the AIMD/RED system is depicted in Fig. 1 [22]. By a suitable control law, we relate the output $q$ with the input $p$, which makes the original open loop systems into a closed-loop control system to achieve asymptotic stability.

\subsection{Stability of heterogeneous AIMD/RED system}

In the previous subsection, we discussed the stability property of the homogeneous-flow system

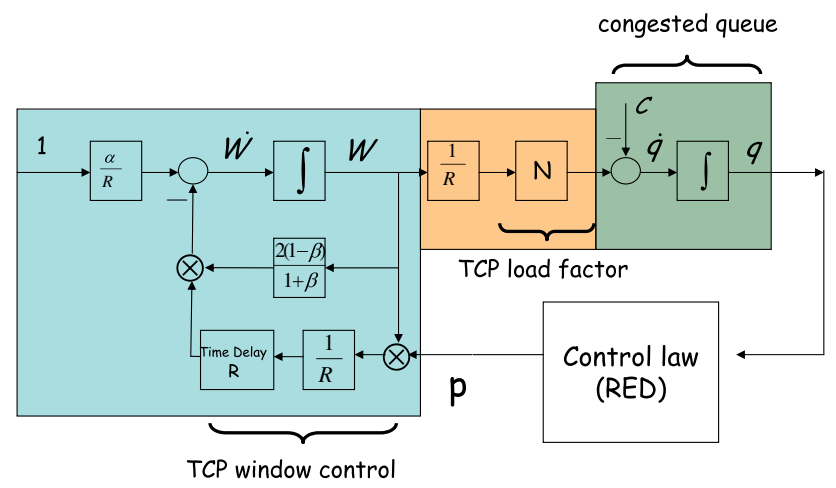

Fig. 1. Block diagram of generalized AIMD/RED system. 
when there is only one type of flows with the parameter pair $(\alpha, \beta)$. To support heterogeneous multimedia applications, we study the system with heterogeneous flows, i.e., there are two or more types of flows with the parameter pairs $\left(\alpha_{1}, \beta_{1}\right)$, $\left(\alpha_{2}, \beta_{2}\right), \ldots,\left(\alpha_{m}, \beta_{m}\right)$. First, we consider the case when there are two different heterogeneous flows: $W_{\mathrm{I}}$ whose $r t t$ is $R_{1}$, and $W_{\mathrm{II}}$ whose $r t t$ is $R_{2}$, with the parameters $\left(\alpha_{1}, \beta_{1}\right),\left(\alpha_{2}, \beta_{2}\right)$, respectively. The number of $W_{\mathrm{I}}$ flows is $N_{1}$, and that of $W_{\mathrm{II}}$ flows is $N_{2}$. Then the corresponding mathematical model has the following form,

$$
\begin{aligned}
& \frac{\mathrm{d} W_{\mathrm{I}}(t)}{\mathrm{d} t}=\frac{\alpha_{1}}{R_{1}}-\frac{2\left(1-\beta_{1}\right)}{1+\beta_{1}} \cdot \frac{W_{\mathrm{I}}^{2}(t)}{R_{1}} \cdot K_{p} q(t), \\
& \frac{\mathrm{d} W_{\mathrm{II}}(t)}{\mathrm{d} t}=\frac{\alpha_{2}}{R_{2}}-\frac{2\left(1-\beta_{2}\right)}{1+\beta_{2}} \cdot \frac{W_{\mathrm{II}}^{2}(t)}{R_{2}} \cdot K_{p} q(t), \\
& \frac{\mathrm{d} q(t)}{\mathrm{d} t}= \begin{cases}\frac{N_{1} W_{\mathrm{I}}(t)}{R_{1}}+\frac{N_{2} W_{\mathrm{II}}(t)}{R_{2}}-C, & q>0, \\
\left\{\frac{N_{1} W_{\mathrm{I}}(t)}{R_{1}}+\frac{N_{2} W_{\mathrm{II}}(t)}{R_{2}}-C\right\}^{+}, & q=0 .\end{cases}
\end{aligned}
$$

The equilibrium points $\left(W_{\mathrm{I}}^{*}, W_{\mathrm{II}}^{*}, q_{0}^{*}\right)$ of (5) can be obtained as

$$
\begin{aligned}
& W_{\mathrm{I}}^{*}=\frac{R_{1} R_{2} C}{R_{2} N_{1}+\left(\frac{\alpha_{2}\left(1-\beta_{1}\right)\left(1+\beta_{2}\right)}{\alpha_{1}\left(1+\beta_{1}\right)\left(1-\beta_{2}\right)}\right)^{1 / 2} \cdot R_{1} N_{2}}, \\
& W_{\mathrm{II}}^{*}=\frac{R_{1} R_{2} C}{\left(\frac{\alpha_{1}\left(1+\beta_{1}\right)\left(1-\beta_{2}\right)}{\alpha_{2}\left(1-\beta_{1}\right)\left(1+\beta_{2}\right)}\right)^{1 / 2} \cdot R_{2} N_{1}+R_{1} N_{2}}, \\
& q_{0}^{*}=\frac{\alpha_{1}\left(1+\beta_{1}\right)\left[R_{2} N_{1}+\left(\frac{\alpha_{2}\left(1-\beta_{1}\right)\left(1+\beta_{2}\right)}{\alpha_{1}\left(1+\beta_{1}\right)\left(1-\beta_{2}\right)}\right)^{1 / 2} R_{1} N_{2}\right]^{2}}{2 R_{1}^{2} R_{2}^{2} C^{2} K_{p}\left(1-\beta_{1}\right)} .
\end{aligned}
$$

With the transformed variables $\widetilde{W}_{\mathrm{I}}(t):=W_{\mathrm{I}}(t)-$ $W_{\mathrm{I}}^{*}, \quad \widetilde{W}_{\mathrm{II}}(t):=W_{\mathrm{II}}(t)-W_{\mathrm{II}}^{*}$ and $\tilde{q}(t):=q(t)-q_{0}^{*}$, (5) becomes

$$
\begin{aligned}
\dot{\widetilde{W}}_{\mathrm{I}}(t)= & -\frac{2\left(1-\beta_{1}\right)}{1+\beta_{1}} \frac{\left(\widetilde{W}_{\mathrm{I}}(t)+W_{\mathrm{I}}^{*}\right)^{2}}{R_{1}} K_{p} \tilde{q}(t) \\
& -\frac{2\left(1-\beta_{1}\right)}{1+\beta_{1}} \frac{\widetilde{W}_{\mathrm{I}}^{2}(t)+2 W_{\mathrm{I}}^{*} \widetilde{W}_{\mathrm{I}}(t)}{R_{1}} K_{p} q_{0}^{*}, \\
\dot{\widetilde{W}}_{\mathrm{II}}(t)= & -\frac{2\left(1-\beta_{2}\right)}{1+\beta_{2}} \frac{\left(\widetilde{W}_{\mathrm{II}}(t)+W_{\mathrm{II}}^{*}\right)^{2}}{R_{2}} K_{p} \tilde{q}(t) \\
& -\frac{2\left(1-\beta_{2}\right)}{1+\beta_{2}} \frac{\widetilde{W}_{\mathrm{II}}^{2}(t)+2 W_{\mathrm{II}}^{*} \widetilde{W}_{\mathrm{II}}(t)}{R_{2}} K_{p} q_{0}^{*}, \\
\dot{\tilde{q}}(t)= & \frac{N_{1} \cdot \widetilde{W}_{\mathrm{I}}(t)}{R_{1}}+\frac{N_{2} \cdot \widetilde{W}_{\mathrm{II}}(t)}{R_{2}} .
\end{aligned}
$$

The equilibrium point of (7) is then $\left(\widetilde{W}_{\mathrm{I}}^{*}, \widetilde{W}_{\mathrm{II}}^{*}\right.$, $\left.\tilde{q}_{0}^{*}\right)=(0,0,0)$.

With (7), choose the following positive-definite Lyapunov function,

$$
\begin{aligned}
& V\left(\widetilde{W}_{\mathrm{I}}(t), \widetilde{W}_{\mathrm{II}}(t), \tilde{q}(t)\right) \\
& =\frac{\left(1+\beta_{1}\right) N_{1}}{2\left(1-\beta_{1}\right) W_{\mathrm{I}}^{* 2}} \cdot \widetilde{W}_{\mathrm{I}}^{2}(t)+\frac{\left(1+\beta_{2}\right) N_{2}}{2\left(1-\beta_{2}\right) W_{\mathrm{II}}^{* 2}} \cdot \widetilde{W}_{\mathrm{II}}^{2}(t)+K_{p} \tilde{q}^{2}(t) .
\end{aligned}
$$

Then,

$$
\begin{aligned}
\dot{V}= & \frac{\left(1+\beta_{1}\right) N_{1}}{\left(1-\beta_{1}\right) W_{\mathrm{I}}^{* 2}} \widetilde{W}_{\mathrm{I}}(t) \dot{\tilde{W}}_{\mathrm{I}}(t) \\
& +\frac{\left(1+\beta_{2}\right) N_{2}}{\left(1-\beta_{2}\right) W_{\mathrm{II}}^{* 2}} \widetilde{W}_{\mathrm{II}}(t) \dot{\widetilde{W}}_{\mathrm{II}}(t)+2 K_{p} \tilde{q}(t) \dot{\tilde{q}}(t) \\
= & -\frac{2 N_{1} K_{p}}{W_{\mathrm{I}}^{* 2} R_{1}} \widetilde{W}_{\mathrm{I}}^{2}(t)\left(\widetilde{W}_{\mathrm{I}}(t)+2 W_{\mathrm{I}}^{*}\right)\left(\tilde{q}(t)+q_{0}^{*}\right) \\
& -\frac{2 N_{2} K_{p}}{W_{\mathrm{II}}^{* 2} R_{2}} \widetilde{W}_{\mathrm{II}}^{2}(t)\left(\widetilde{W}_{\mathrm{II}}(t)+2 W_{\mathrm{II}}^{*}\right)\left(\tilde{q}(t)+q_{0}^{*}\right) \leqslant 0 .
\end{aligned}
$$

From the physics constraint point of view, the positive-definite Lyapunov function emulates the total energy function of the system, i.e., the sum of kinetic and potential energy. Here $\dot{V} \leqslant 0$, since $\widetilde{W}_{\mathrm{I}}(t)+2 W_{\mathrm{I}}^{*}>0, \quad \widetilde{W}_{\mathrm{II}}(t)+2 W_{\mathrm{II}}^{*}>0$ and $\tilde{q}(t)+$ $q_{0}^{*} \geqslant 0$, which means the energy of the system is non-increasing. Thus, we prove that the equilibrium point is stable. To conclude asymptotic stability, we first consider the set of states where $\dot{V}=0$,

$$
\begin{aligned}
\mathscr{M} & :=\left\{\left(\widetilde{W}_{\mathrm{I}}, \widetilde{W}_{\mathrm{II}}, \tilde{q}\right): \dot{V}=0\right\} \\
& =\left\{\left(\widetilde{W}_{\mathrm{I}}, \widetilde{W}_{\mathrm{II}}, \tilde{q}\right): \widetilde{W}_{\mathrm{I}}=\widetilde{W}_{\mathrm{II}}=0 \text { or } \tilde{q}=-q_{0}^{*}\right\} .
\end{aligned}
$$

By LaSalle's Invariance Principle [24], trajectories of (7) converge to the largest invariant set contained in $\mathscr{M}$. We will then prove that the only invariant set contained in $\mathscr{M}$ is the equilibrium point $(0,0,0)$. If $\left(\widetilde{W}_{\mathrm{I}}, \widetilde{W}_{\mathrm{II}}, \tilde{q}\right)$ is equal to $(0,0, \tilde{q})$ or $\left(\widetilde{W}_{\mathrm{I}}, \widetilde{W}_{\mathrm{II}},-q_{0}^{*}\right)$, by using (7), we can conclude that $\left(\widetilde{W}_{\mathrm{I}}\left(t^{+}\right)\right.$, $\left.\widetilde{W}_{\text {II }}\left(t^{+}\right), \tilde{q}\left(t^{+}\right)\right)$is not in $\mathscr{M}$, which implies that no trajectory can stay in $\mathscr{M}$, other than the point $(0,0,0)$. Therefore, asymptotic stability is obtained, which we summarize as follows:

Theorem 2. For any $K_{p}>0$, the equilibrium point of (7) is asymptotically stable for any positive pairs $\left(\alpha_{1}, \beta_{1}\right),\left(\alpha_{2}, \beta_{2}\right)$ and any positive $R_{1}$ and $R_{2}$.

We can also extend our results to the case when more than two heterogeneous flows exist in the same system. Suppose that there are $M$ different heterogeneous flows $\left(\alpha_{1}, \beta_{1}\right),\left(\alpha_{2}, \beta_{2}\right), \ldots,\left(\alpha_{m}, \beta_{m}\right)$ sharing the 
resources, with the number $N_{1}, N_{2}, \ldots, N_{m}$, and different $r t t s R_{1}, R_{2}, \ldots, R_{m}$ respectively, then those flows can be mathematically modeled as

$$
\begin{aligned}
& \frac{\mathrm{d} W_{\mathrm{I}}(t)}{\mathrm{d} t}=\frac{\alpha_{1}}{R_{1}}-\frac{2\left(1-\beta_{1}\right)}{1+\beta_{1}} \cdot \frac{W_{\mathrm{I}}(t)^{2}}{R_{1}} \cdot K_{p} q(t), \\
& \frac{\mathrm{d} W_{\mathrm{II}}(t)}{\mathrm{d} t}=\frac{\alpha_{2}}{R_{2}}-\frac{2\left(1-\beta_{2}\right)}{1+\beta_{2}} \cdot \frac{W_{\mathrm{II}}(t)^{2}}{R_{2}} \cdot K_{p} q(t), \\
& \frac{\mathrm{d} W_{M}(t)}{\mathrm{d} t}=\frac{\alpha_{m}}{R_{m}}-\frac{2\left(1-\beta_{m}\right)}{1+\beta_{m}} \cdot \frac{W_{M}(t)^{2}}{R_{m}} \cdot K_{p} q(t), \\
& \frac{\mathrm{d} q(t)}{\mathrm{d} t}=\left\{\begin{array}{l}
\sum_{i=1}^{m} \frac{N_{i} W_{i}(t)}{R_{i}}-C, \\
\left\{\sum_{i=1}^{m} \frac{N_{i} W_{i}(t)}{R_{i}}-C\right\}^{+}, \quad q=0 .
\end{array}\right.
\end{aligned}
$$

With (8), we choose a positive-definite Lyapunov function as

$$
\begin{aligned}
& V\left(\widetilde{W}_{\mathrm{I}}(t), \widetilde{W}_{\mathrm{II}}(t), \ldots, \widetilde{W}_{M}(t), \tilde{q}(t)\right) \\
& =\frac{\left(1+\beta_{1}\right) N_{1}}{2\left(1-\beta_{1}\right) W_{\mathrm{I}}^{* 2}} \cdot \widetilde{W}_{\mathrm{I}}^{2}(t)+\frac{\left(1+\beta_{2}\right) N_{1}}{2\left(1-\beta_{2}\right) W_{\mathrm{II}}^{* 2}} \cdot \widetilde{W}_{\mathrm{II}}^{2}(t) \\
& \quad+\cdots+\frac{\left(1+\beta_{m}\right) N_{m}}{2\left(1-\beta_{m}\right) W_{M}^{* 2}} \cdot \widetilde{W}_{M}^{2}(t)+K_{p} \tilde{q}^{2}(t),
\end{aligned}
$$

where $\widetilde{W}_{i}(t), i=1,2, \ldots, m$, and $\tilde{q}(t)$ have the same meaning as in (7). Then,

$$
\begin{aligned}
\dot{V}= & \frac{\left(1+\beta_{1}\right) N_{1}}{\left(1-\beta_{1}\right) W_{\mathrm{I}}^{* 2}} \widetilde{W}_{\mathrm{I}} \dot{\tilde{W}}_{\mathrm{I}}+\frac{\left(1+\beta_{2}\right) N_{2}}{\left(1-\beta_{2}\right) W_{\mathrm{II}}^{* 2}} \widetilde{W}_{\mathrm{II}} \dot{\tilde{W}}_{\mathrm{II}} \\
& +\cdots+\frac{\left(1+\beta_{m}\right) N_{M}}{\left(1-\beta_{m}\right) \widetilde{W}_{M}^{* 2}} \widetilde{W}_{M} \dot{\tilde{W}}_{M}+2 K_{p} \tilde{q} \dot{\tilde{q}} \\
= & -\frac{2 N_{1} K_{p}}{W_{\mathrm{I}}^{* 2} R_{1}} \widetilde{W}_{\mathrm{I}}^{2}\left(\widetilde{W}_{\mathrm{I}}+2 W_{\mathrm{I}}^{*}\right)\left(\tilde{q}+q_{0}^{*}\right)-\cdots \\
& -\frac{2 N_{m} K_{p}}{W_{M}^{* 2} R_{m}} \widetilde{W}_{M}^{2}\left(\widetilde{W}_{M}+2 W_{M}^{*}\right)\left(\tilde{q}+q_{0}^{*}\right) \leqslant 0 .
\end{aligned}
$$

We can obtain its asymptotic stability by applying LaSalle's Invariance Principle, and thus have the following theorem,

Theorem 3. For any $K_{p}>0$, the equilibrium point of system (8) is asymptotically stable for any positive pairs $\left(\alpha_{1}, \beta_{1}\right),\left(\alpha_{2}, \beta_{2}\right), \ldots,\left(\alpha_{m}, \beta_{m}\right)$ and any positive $R_{1}, R_{2}, \ldots, R_{m}$.

\subsection{TCP-friendliness and differentiated services}

For two competing AIMD flows, from (6), we can also get the relationship between $W_{\mathrm{I}}^{*}$ and $W_{\mathrm{II}}^{*}$ as follows:

$$
\frac{W_{I}^{*}}{W_{\mathrm{II}}^{*}}=\left[\frac{\alpha_{1}\left(1+\beta_{1}\right)\left(1-\beta_{2}\right)}{\alpha_{2}\left(1-\beta_{1}\right)\left(1+\beta_{2}\right)}\right]^{1 / 2} .
$$

This means that the ratio of $W_{\mathrm{I}}^{*}$ and $W_{\mathrm{II}}^{*}$ depends only on the choices of $\left(\alpha_{1}, \beta_{1}\right)$ and $\left(\alpha_{2}, \beta_{2}\right)$, and regardless of the traffic loads in the network and their initial states. Therefore, by choosing suitable $\left(\alpha_{1}, \beta_{1}\right)$ and $\left(\alpha_{2}, \beta_{2}\right)$, we can guarantee the fair share of bottleneck bandwidth for each flow. Consequently, for $\operatorname{AIMD}(\alpha, \beta)$ flows to be TCP-friendly, i.e., co-existing TCP and AIMD flows obtain the same share of bottleneck bandwidth, the necessary and sufficient condition is

$\alpha=\frac{3(1-\beta)}{1+\beta}$.

A large value of $\beta$ can be chosen for applications that cannot tolerate drastic changes of the throughput, and $\alpha$ can be set according to the TCP-friendly condition.

In the Internet, different types of multimedia services are provided with different resource requirements. To provide differentiate services, we can assign different traffic a different weight. Eq. (9) indicates that we can easily adjust the AIMD parameters of the end systems to provide differentiated services according to different QoS requirements. For instance, let the throughput of an $\operatorname{AIMD}\left(\alpha_{1}, \beta_{1}\right)$ flow be $k$ times that of an AIM$\mathrm{D}\left(\alpha_{2}, \beta_{2}\right)$ flow, the AIMD parameter pairs should satisfy

$\frac{\alpha_{1}}{\alpha_{2}}=\frac{k^{2}\left(1-\beta_{1}\right)\left(1+\beta_{2}\right)}{\left(1+\beta_{1}\right)\left(1-\beta_{2}\right)}$.

\subsection{Numeric results}

The traces of average window size and queue length of 100 TCP $(\alpha=1, \beta=0.5)$ flows and 100 $\operatorname{AIMD}(0.2,0.875)$ flows are given in Figs. 2 and 3, respectively. The parameters used are $C=$ $100,000 \mathrm{packet} / \mathrm{s}, \quad R=100 \mathrm{~ms}, \quad K_{p}=0.0001$, and $\min _{\text {th }}=200$ packets. For the TCP-friendliness, let 100 TCP flows and $24 \operatorname{AIMD}(0.2,0.875)$ flows share the bottleneck, and the numeric results with Matlab are shown in Fig. 4. It can be seen that when the flows in the network possess the same $(\alpha, \beta)$ parameter pair, the ensemble averages of window size and the bottleneck queue length converge to some certain values, i.e., the equilibrium points we derived 


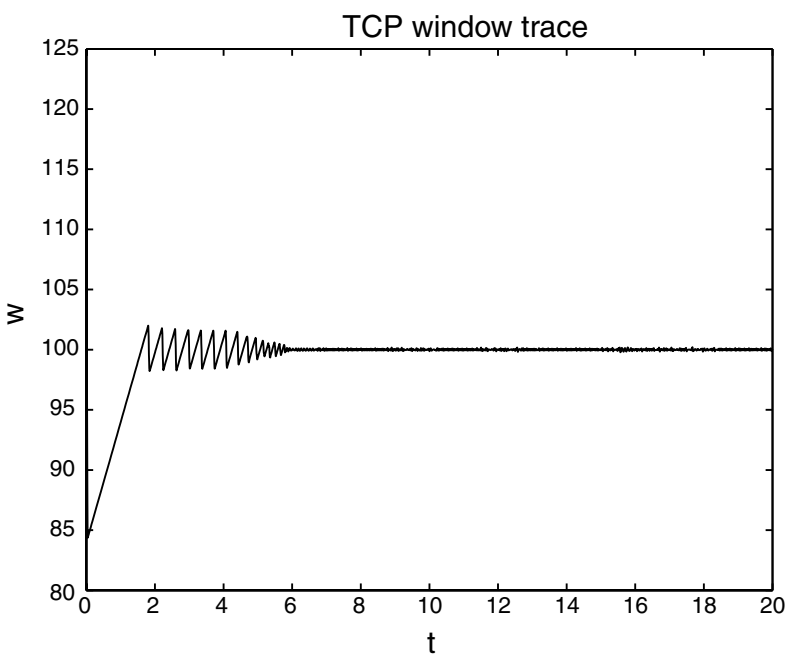

(a) TCP window trace

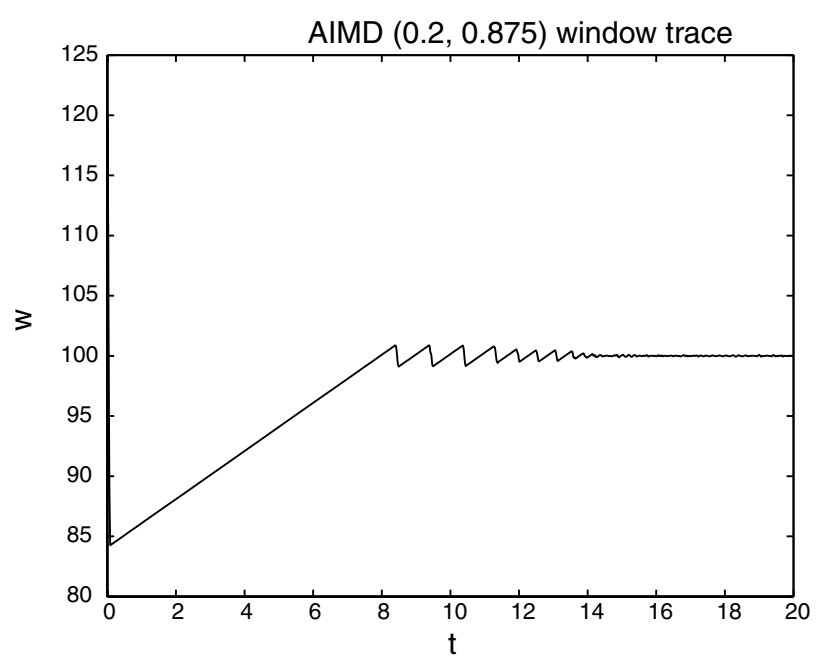

(b) $\operatorname{AIMD}(0.2,0.875)$ window trace

Fig. 2. Window trace.

in the previous analysis. When TCP and $\operatorname{AIMD}(0.2,0.875)$ flows co-exist, they will fairly share the link capacity in steady state, since $(0.2,0.875)$ satisfies the TCP-friendly condition (10). Thus, the numeric results validate the theorems.

Furthermore, from Figs. 2 and 3, with a smaller value of $\alpha$ and a larger value of $\beta$, it takes longer time for the system to converge to the steady state, and the link utilization during the transient stage is low; however, in steady state, the oscillation amplitudes of the instantaneous window size and queue length are smaller. In other words, with a smaller value of $\alpha$ and a larger value of $\beta$, the queuing delay jitter is smaller, and the link utilization in steady state is higher, which are desired for supporting time-sensitive multimedia applications.

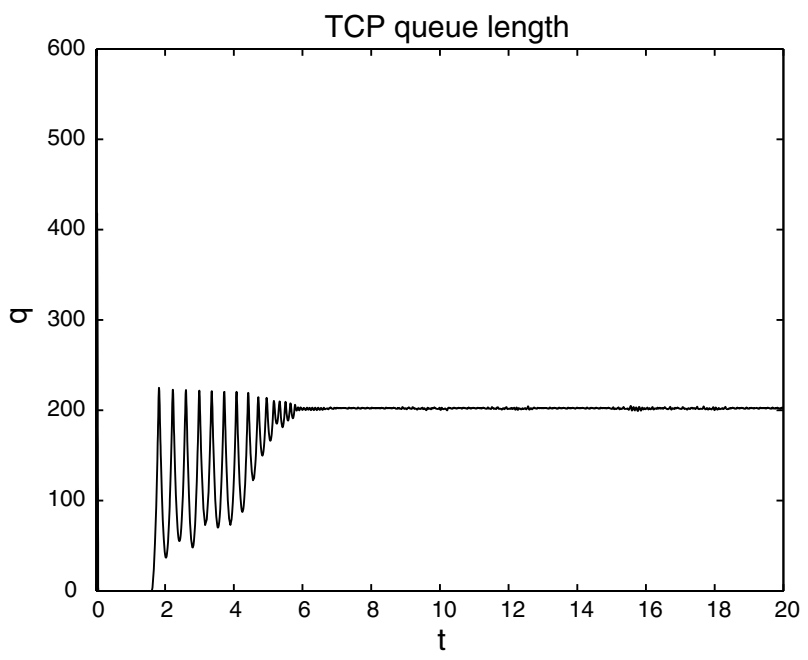

(a) TCP queue length

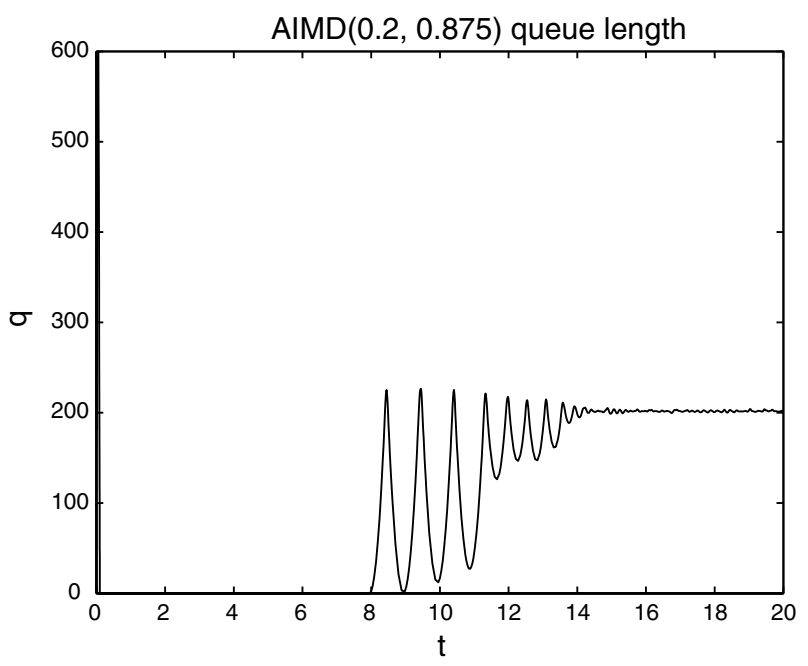

(b) $\operatorname{AIMD}(0.2,0.875)$ queue length

Fig. 3. Queue length.

\section{Stability and fairness analysis with heterogeneous feedback delays}

In this section, we study the stability properties of the AIMD/RED system with feedback delay, using the method of Lyapunov functional and Lyapunov function with Lyapunov-Razumikhin condition, to establish different sufficient conditions for the stability of the AIMD/RED system with heterogeneous flows and feedback delays.

\subsection{Stability of homogeneous delayed AIMD/RED system}

For AIMD/RED system with feedback delay, i.e., $p(t-R(t))=K_{p} q(t-R(t))$, we can obtain the equilibrium point $\left(W_{0}^{*}, q_{0}^{*}\right)$ of the system (1) as 


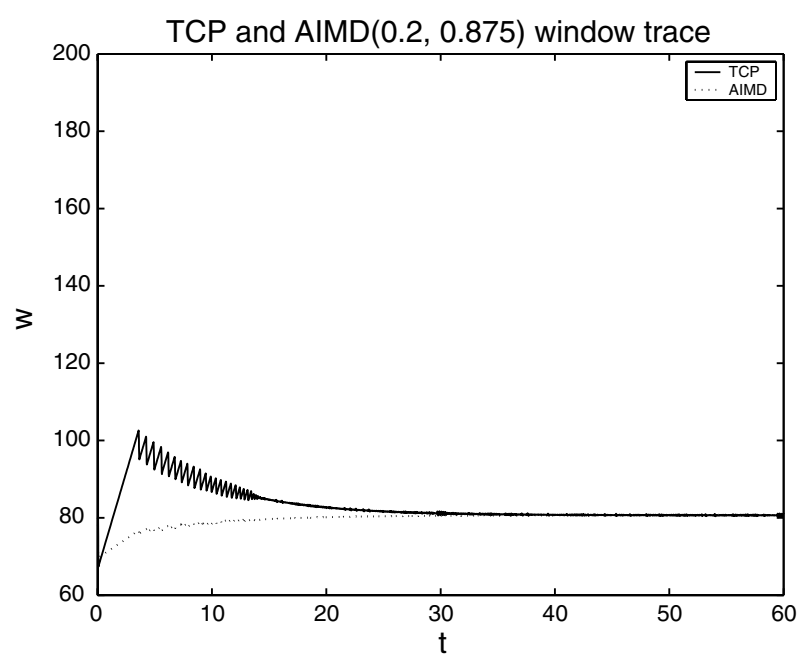

(a) TCP and $\operatorname{AIMD}(0.2,0.875)$ window trace

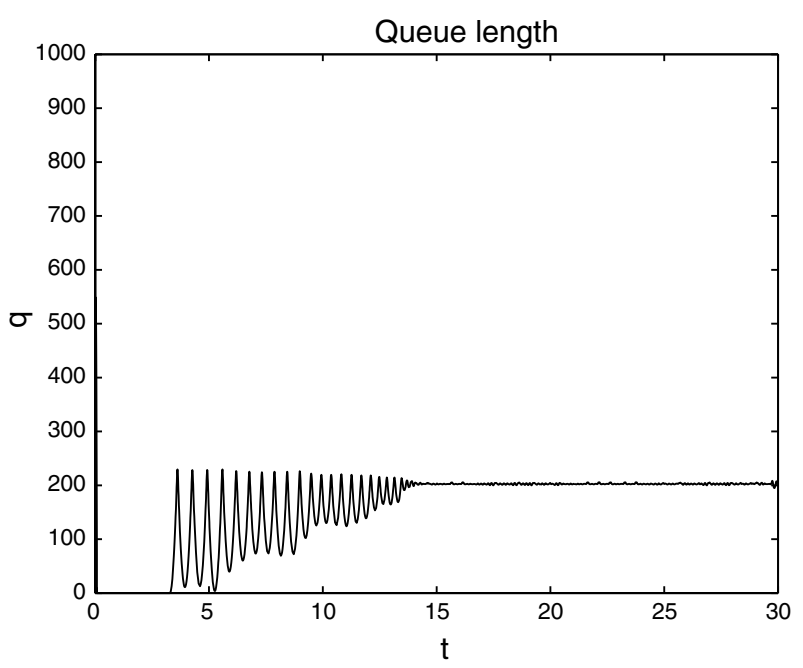

(b) Queue length

Fig. 4. TCP-friendliness.

$W_{0}^{*}=\frac{R^{*} C}{N} ; \quad q_{0}^{*}=\frac{\alpha(1+\beta) N^{2}}{2(1-\beta) R^{* 2} C^{2} K_{p}}$,

where $R^{*}=\frac{q_{0}^{*}}{C}+T_{p}$. Due to the highly nonlinear nature and the effect of delays in the system, no suitable Lyapunov function could be constructed to prove global asymptotic stability of the equilibrium. Without loss of generality, we ignore the dependence of the time-delay argument $t-R(t)$ on the queuing delay and assume it is fixed to $t-R^{*}$. Then, the system (2) can be linearized as

$$
\begin{aligned}
\dot{\tilde{W}}(t)= & -\frac{\alpha N}{R^{* 2} C} \widetilde{W}(t)-\frac{\alpha N}{R^{* 2} C} \widetilde{W}\left(t-R^{*}\right)-\frac{\alpha}{R^{* 2} C} \tilde{q}(t) \\
& -\left(\frac{2(1-\beta)}{1+\beta} \frac{K_{p} C^{2} R^{*}}{N^{2}}-\frac{\alpha}{R^{* 2} C}\right) \tilde{q}\left(t-R^{*}\right), \\
\dot{\tilde{q}}(t)= & \frac{N}{R^{*}} \widetilde{W}(t)-\frac{1}{R^{*}} \tilde{q}(t),
\end{aligned}
$$

where $\widetilde{W}:=W-W_{0}^{*}, \tilde{q}:=q-q_{0}^{*}$.

System (13) can be written in the form of

$\dot{x}(t)=A x(t)+B x\left(t-R^{*}\right)$,

with $x=(\widetilde{W}(t), \tilde{q}(t))^{\mathrm{T}}, A=\left[\begin{array}{cc}\frac{-\alpha N}{R^{* 2} C} & \frac{-\alpha}{R^{2} C} \\ \frac{N}{R^{*}} & -\frac{1}{R^{*}}\end{array}\right]$ and

$B=\left[\begin{array}{cc}\frac{-\alpha N}{R^{* 2} C} & \frac{-2(1-\beta)}{1+\beta} \frac{K_{p} C^{2} R^{*}}{N^{2}}+\frac{\alpha}{R^{* 2} C} \\ 0 & 0\end{array}\right]$.

The norm of matrix is defined by $\|A\|=$ $\sqrt{\lambda_{\max }\left(A^{\mathrm{T}} A\right)}$, i.e., the square root of the maximum eigenvalue of $A^{\mathrm{T}} A$.

It can be checked that $A$ is a Hurwitz matrix, which implies that for any positive-definite matrix $Q$, there exists certain positive-definite matrix $P$, such that $A^{\mathrm{T}} P+P A=-Q$.

Theorem 4. Let $M=\sqrt{\lambda_{\max }(P) / \lambda_{\min }(P)}$, if there exist positive-definite $P$ and $Q$ satisfying $A^{\mathrm{T}} P+$ $P A=-Q$ such that matrix $Q-2 M\|P B\| I$ is positive definite, then the equilibrium point of (2) is locally asymptotically stable.

Proof. With (13) and (14), we choose Lyapunov function $V(x)=x^{\mathrm{T}} P x$. Then

$$
\begin{aligned}
\dot{V} & =\dot{x}^{\mathrm{T}} P x+x^{\mathrm{T}} P \dot{x} \\
& =x^{\mathrm{T}}(t)\left(A^{\mathrm{T}} P+P A\right) x(t)+2 x^{\mathrm{T}}\left(t-R^{*}\right) B^{\mathrm{T}} P x(t) .
\end{aligned}
$$

Applying Lyapunov-Razumikhin condition, we assume $\mu>1$ such that

$$
V(\xi) \leqslant \mu^{2} V(t) \text { for } t-R^{*} \leqslant \xi \leqslant t,
$$

which implies that $\|x(\xi)\| \leqslant M \cdot \mu \cdot\|x(t)\|$, where $M=\sqrt{\frac{\lambda_{\max }(P)}{\lambda_{\min }(P)}}$.

Thus,

$$
\begin{aligned}
\dot{V} & \leqslant-x^{\mathrm{T}}(t) Q x(t)+2\left\|x\left(t-R^{*}\right)\right\|\|P B\|\|x(t)\| \\
& \leqslant-x^{\mathrm{T}}(t)[Q-2 \mu M\|P B\| I] x(t) .
\end{aligned}
$$

Since $Q-2 M\|P B\| I$ is positive definite, there exists $\mu>1$ such that $\dot{V}<0$. The local asymptotic stability of system (2) is then obtained.

Lyapunov-Razumikhin condition is used in Theorem 4 to deal with the delayed terms in $\dot{V}$. Lyapunov functional is another method that can be applied when studying the stability of delayed systems. In the following, we apply the method of Lyapunov functional to give a different sufficient condition for the local asymptotic stability of system (2).

Theorem 5. If there exist positive-definite $P$ and $Q$ satisfying $A^{T} P+P A=-Q$ and positive-definite $H$ 
such that matrix $\left[\begin{array}{cc}Q-H & -P B \\ -B^{T} P & H\end{array}\right]$ is positive definite, the equilibrium point of (2) is locally asymptotically stable.

Proof. With (13) and (14), we choose Lyapunov functional $V(x)=x^{\mathrm{T}} P x+\int_{t-R^{*}}^{t} x^{\mathrm{T}}(s) H x(s) \mathrm{d} s$, then

$$
\begin{aligned}
\dot{V}= & x^{\mathrm{T}}(t)\left(A^{\mathrm{T}} P+P A\right) x(t)+2 x^{\mathrm{T}}\left(t-R^{*}\right) B^{\mathrm{T}} P x(t) \\
& +x^{\mathrm{T}}(t) H x(t)-x^{\mathrm{T}}\left(t-R^{*}\right) H x\left(t-R^{*}\right) \\
= & -x^{\mathrm{T}}(t)(Q-H) x(t)+2 x^{\mathrm{T}}\left(t-R^{*}\right) B^{\mathrm{T}} P x(t) \\
& -x^{\mathrm{T}}\left(t-R^{*}\right) H x\left(t-R^{*}\right) \\
= & -\left(x^{\mathrm{T}}(t), x^{\mathrm{T}}\left(t-R^{*}\right)\right)\left[\begin{array}{cc}
Q-H & -P B \\
-B^{\mathrm{T}} P & H
\end{array}\right]\left[\begin{array}{c}
x(t) \\
x\left(t-R^{*}\right)
\end{array}\right] .
\end{aligned}
$$

Thus, system (2) is locally asymptotically stable if $\left[\begin{array}{cc}Q-H & -P B \\ -B^{\mathrm{T}} P & H\end{array}\right]$ is positive definite.

The two theorems provide sufficient conditions of local asymptotic stability for the AIMD/RED system. We give a numerical example for Theorem 5: Let $N=10, \quad C=3000$ (packets $/ \mathrm{s}), \quad T_{p}=0.02(\mathrm{~s})$, $K_{p}=0.0005$ with $\alpha=1, \quad \beta=0.5$. We choose $Q=\left[\begin{array}{cc}39.0410 & 2.2648 \\ 2.2648 & 6.4539\end{array}\right]$ and $H=\frac{1}{2} Q$. Note that $Q$ and $H$ are positive definite. With Matlab, we get $P=\left[\begin{array}{cc}19.0990 & 0.2793 \\ 0.2793 & 0.0599\end{array}\right]$ and the eigenvalues of the matrix $\left[\begin{array}{cc}Q-H & -P B \\ -B^{\mathrm{T}} P & H\end{array}\right]$ are all positive: 0.1780 , 3.2305, 3.4105, 38.6758; therefore, $\left[\begin{array}{cc}Q-H & -P B \\ -B^{\mathrm{T}} P & H\end{array}\right]$ is positive definite. Thus, the condition of Theorem 5 holds and the system is locally asymptotically stable. Simulation results using the same parameters will be given in Section 6 .

Theorems 4 and 5 give different sufficient asymptotic stability conditions, which allow us to use any of them at our convenience. Again, the asymptotic stability is for the average values of window size and queue length. Given that the average window size converges to $W_{0}^{*}$, the maximum instantaneous window size is bounded to $2 W_{0}^{*} /(1+\beta)$, so the AIMD window size can be marginally stable with known bounds. Similarly, the instantaneous queue length is bounded.

So far, we have mathematically derived the local stability conditions of AIMD/RED system. For local asymptotic stability, once the system enters the stability region or region-of-attraction, the system will converge to the equilibrium asymptotically. Obviously, the equilibrium point belongs to the stability region. We conjecture that, with both the slow-start and the AIMD algorithms of the TCP/AIMD protocols, the system will eventually evolve to the stability region and equilibrium, and thus global asymptotic stability can be achieved. Simulations in Section 6 also demonstrate this tendency. Global asymptotic stability conditions for AIMD/RED systems are still under investigation.

\subsection{Stability of heterogeneous delayed AIMD/RED system}

In the previous subsection, we discuss the stability issue of homogeneous flows with the same $\operatorname{AIMD}(\alpha, \beta)$ pair and the same round-trip delay. In this section, we first consider two classes of flows with parameters $\left(\alpha_{1}, \beta_{1}\right),\left(\alpha_{2}, \beta_{2}\right)$, traffic loads $N_{1}, N_{2}$ and rtts $R_{1}, R_{2}$, respectively. The results can be generalized to any number of flows with heterogeneous AIMD parameters and feedback delays.

Taking all the time delays into consideration, the AIMD/RED system shared by two classes of flows can be modeled as

$$
\begin{aligned}
& \frac{\mathrm{d} W_{\mathrm{I}}(t)}{\mathrm{d} t}= \frac{\alpha_{1}}{R_{1}(t)}-\frac{2\left(1-\beta_{1}\right)}{1+\beta_{1}} \\
& \times \frac{W_{\mathrm{I}}(t) W_{\mathrm{I}}\left(t-R_{1}(t)\right)}{R_{1}\left(t-R_{1}(t)\right)} K_{p} q\left(t-R_{1}\right), \\
& \frac{\mathrm{d} W_{\mathrm{II}}(t)}{\mathrm{d} t}= \frac{\alpha_{2}}{R_{2}(t)}-\frac{2\left(1-\beta_{2}\right)}{1+\beta_{2}} \\
& \times \frac{W_{\mathrm{II}}(t) W_{\mathrm{II}}\left(t-R_{2}(t)\right)}{R_{2}\left(t-R_{2}(t)\right)} K_{p} q\left(t-R_{2}\right), \\
& \frac{\mathrm{d} q(t)}{\mathrm{d} t}=\left\{\begin{array}{l}
\frac{N_{1} W_{\mathrm{I}}(t)}{R_{1}(t)}+\frac{N_{2} W_{\mathrm{II}}(t)}{R_{2}(t)}-C, \\
\left\{\frac{N_{1} W_{\mathrm{I}}(t)}{R_{1}(t)}+\frac{N_{2} W_{\mathrm{II}}(t)}{R_{2}(t)}-C\right\}^{+}, \quad q=0,
\end{array}\right.
\end{aligned}
$$

with $R_{1}(t)=\frac{q(t)}{C}+T_{p 1} R_{2}(t)=\frac{q(t)}{C}+T_{p 2}$.

Then, the delayed linearized system about the equilibrium point is 


$$
\begin{aligned}
\dot{\widetilde{W}}_{\mathrm{I}}(t)= & -\frac{\alpha_{1}\left(N_{1} R_{2}^{*} G+N_{2} R_{1}^{*}\right)}{G C R_{1}^{*} R_{2}^{*}}\left(\widetilde{W}_{\mathrm{I}}(t)-\widetilde{W}_{\mathrm{I}}\left(t-R_{1}^{*}\right)\right) \\
& -\frac{2\left(1-\beta_{1}\right)}{1+\beta_{1}} \frac{K_{p} G^{2} C^{2} R_{1}^{*} R_{2}^{* 2}}{\left(N_{1} R_{2}^{*} G+N_{2} R_{1}^{*}\right)^{2}} \tilde{q}\left(t-R_{1}^{*}\right) \\
& -\frac{\alpha_{1} \tilde{q}(t)}{R_{1}^{* 2} C}+\frac{\alpha_{1}}{C R_{1}^{* 2}} \tilde{q}\left(t-R_{1}^{*}\right), \\
\dot{\widetilde{W}}_{\mathrm{II}}(t)= & -\frac{\alpha_{2}\left(N_{1} R_{2}^{*} G+N_{2} R_{1}^{*}\right)}{C R_{1}^{*} R_{2}^{*}} \\
& \times\left(\widetilde{W}_{\mathrm{II}}(t)-\widetilde{W}_{\mathrm{II}}\left(t-R_{2}^{*}\right)\right) \\
& -\frac{2\left(1-\beta_{2}\right)}{1+\beta_{2}} \frac{K_{p} C^{2} R_{1}^{* 2} R_{2}}{\left(N_{1} R_{2}^{*} G+N_{2} R_{1}^{*}\right)^{2}} \tilde{q}\left(t-R_{2}^{*}\right) \\
& -\frac{\alpha_{2} \tilde{q}(t)}{R_{2}^{* 2} C}+\frac{\alpha_{2}}{C R_{2}^{* 2}} \tilde{q}\left(t-R_{2}^{*}\right), \\
\dot{\tilde{q}}(t)= & \frac{N_{1}}{R_{1}^{*}} \widetilde{W}_{\mathrm{I}}(t)+\frac{N_{2}}{R_{2}^{*}} \widetilde{W}_{\mathrm{II}}(t) \\
& -\frac{G N_{1} R_{2}^{*}}{R_{1}^{*}\left(N_{1} R_{2}^{*} G+N_{2} R_{1}^{*}\right)} \tilde{q}(t) \\
& -\frac{N_{2} R_{1}^{*}}{R_{2}^{*}\left(N_{1} R_{2}^{*} G+N_{2} R_{1}^{*}\right)} \tilde{q}(t),
\end{aligned}
$$

where $\widetilde{W}_{\mathrm{I}}:=W-W_{\mathrm{I}}^{*}, \widetilde{W}_{\mathrm{II}}:=W-W_{\mathrm{II}}^{*}, \tilde{q}:=q-q_{0}^{*}$.

$$
\begin{aligned}
& \left(W_{\mathrm{I}}^{*}, W_{\mathrm{II}}^{*}, q_{0}^{*}\right) \\
& \quad=\left(\frac{G C R_{1}^{*} R_{2}^{*}}{N_{1} R_{2}^{*} G+N_{2} R_{1}^{*}}, \frac{C R_{1}^{*} R_{2}^{*}}{N_{1} R_{2}^{*} G+N_{2} R_{1}^{*}}, \frac{\alpha_{1}\left(1+\beta_{1}\right)}{2\left(1-\beta_{1}\right) W_{\mathrm{I}}^{* 2} K_{p}}\right)
\end{aligned}
$$

is the equilibrium point of system (15), where $R_{1}^{*}=\frac{q_{0}^{*}}{C}+T_{p 1}, R_{2}^{*}=\frac{q_{0}^{*}}{C}+T_{p 2}$, and $G=\left(\frac{\alpha_{1}\left(1+\beta_{1}\right)\left(1-\beta_{2}\right)}{\alpha_{2}\left(1-\beta_{1}\right)\left(1+\beta_{2}\right)}\right)^{1 / 2}$.

System (16) can be rewritten as

$\dot{x}(t)=A x(t)+B_{1} x\left(t-R_{1}^{*}\right)+B_{2} x\left(t-R_{2}^{*}\right)$,

with $x=\left(\widetilde{W}_{\mathrm{I}}(t), \widetilde{W}_{\mathrm{II}}(t), \tilde{q}(t)\right)^{\mathrm{T}}$,

$$
\begin{aligned}
& A=\left[\begin{array}{ccc}
a_{11} & 0 & \frac{-\alpha_{1}}{R_{1}^{* 2} C} \\
0 & a_{22} & \frac{-\alpha_{2}}{R_{2}^{* 2} C} \\
\frac{N_{1}}{R_{1}^{*}} & \frac{N_{2}}{R_{2}^{*}} & a_{33}
\end{array}\right], B_{1}=\left[\begin{array}{ccc}
b 1_{11} & 0 & b 1_{13} \\
0 & 0 & 0 \\
0 & 0 & 0
\end{array}\right] \text { and } \\
& B_{2}=\left[\begin{array}{ccc}
0 & 0 & 0 \\
0 & b 2_{22} & b 2_{23} \\
0 & 0 & 0
\end{array}\right] .
\end{aligned}
$$

where

$$
\begin{aligned}
& a_{11}=-\frac{\alpha_{1}\left(N_{1} R_{2}^{*} G+N_{2} R_{1}^{*}\right)}{G C R_{1}^{*} R_{2}^{*}}, \\
& a_{22}=-\frac{\alpha_{2}\left(N_{1} R_{2}^{*} G+N_{2} R_{1}^{*}\right)}{C R_{1}^{*} R_{2}^{*}}, \\
& a_{33}=-\frac{G N_{1} R_{2}^{* 2}+N_{2} R_{1}^{* 2}}{R_{1}^{*} R_{2}^{*}\left(N_{1} R_{2}^{*} G+N_{2} R_{1}^{*}\right)}, \\
& b 1_{11}=-\frac{\alpha_{1}\left(N_{1} R_{2}^{*} G+N_{2} R_{1}^{*}\right)}{G C R_{1}^{*} R_{2}^{*}}, \\
& b 1_{13}=-\frac{2\left(1-\beta_{1}\right)}{1+\beta_{1}} \frac{K_{p} G^{2} C^{2} R_{1}^{*} R_{2}^{* 2}}{\left(N_{1} R_{2}^{*} G+N_{2} R_{1}^{*}\right)^{2}}+\frac{\alpha_{1}}{C R_{1}^{* 2}}, \\
& b 2_{22}=-\frac{\alpha_{2}\left(N_{1} R_{2}^{*} G+N_{2} R_{1}^{*}\right)}{C R_{1}^{*} R_{2}^{*}}, \\
& b 2_{23}=-\frac{2\left(1-\beta_{2}\right)}{1+\beta_{2}} \frac{K_{p} C^{2} R_{1}^{* 2} R_{2}}{\left(N_{1} R_{2}^{*} G+N_{2} R_{1}^{*}\right)^{2}}+\frac{\alpha_{2}}{C R_{2}^{* 2}} .
\end{aligned}
$$

Also, we can check that $A$ is a Hurwitz matrix. Let $M=\sqrt{\lambda_{\max }(P) / \lambda_{\min }(P)}$, we have the following theorem.

Theorem 6. If there exist positive-definite $P$ and $Q$ satisfying $A^{\mathrm{T}} P+P A=-Q$ such that matrix $Q-2 M\left(\left\|P B_{1}\right\|+\left\|P B_{2}\right\|\right) I$ is positive definite, then the equilibrium point of (15) is locally asymptotically stable.

Proof. With (16) and (17), we choose Lyapunov function $V(x)=x^{\mathrm{T}} P x$, then

$$
\begin{aligned}
\dot{V}= & x^{\mathrm{T}}(t)\left(A^{\mathrm{T}} P+P A\right) x(t)+2 x^{\mathrm{T}}\left(t-R_{1}^{*}\right) B_{1}^{\mathrm{T}} P x(t) \\
& +2 x^{\mathrm{T}}\left(t-R_{2}^{*}\right) B_{2}^{\mathrm{T}} P x(t) .
\end{aligned}
$$

Let $R^{*}=\max \left\{R_{1}^{*}, R_{2}^{*}\right\}$. Applying the LyapunovRazumikhin condition, we assume $\mu>1$ such that

$V(\xi) \leqslant \mu^{2} V(t), \quad t-R^{*} \leqslant \xi \leqslant t$,

which implies that $\|x(\xi)\| \leqslant M \cdot \mu \cdot\|x(t)\|$.

Thus,

$$
\begin{aligned}
\dot{V} \leqslant & -x^{\mathrm{T}}(t) Q x(t)+2\left\|x\left(t-R^{*}\right)\right\|\left\|P B_{1}\right\|\|x(t)\| \\
& +2\left\|x\left(t-R^{*}\right)\right\|\left\|P B_{2}\right\|\|x(t)\| \\
\leqslant & -x^{\mathrm{T}}(t)\left[Q-2 \mu M\left(\left\|P B_{1}\right\|+\left\|P B_{2}\right\|\right) I\right] x(t) .
\end{aligned}
$$

Therefore, there exists $\mu>1$ such that $\dot{V}<0$ under the condition of the Theorem. The local asymptotic stability of system (15) is then obtained. 
We can also apply the method of Lyapunov functional to obtain a different sufficient condition for the local asymptotic stability of system (15).

Theorem 7. If there exist positive-definite $P$ and $Q$ satisfying $A^{\mathrm{T}} P+P A=-Q$ and positive-definite $H$ such that matrix $\left[\begin{array}{ccc}Q-2 H & -P B_{1} & -P B_{2} \\ -B_{1}^{T} P & H & 0 \\ -B_{2}^{T} P & 0 & H\end{array}\right]$ is positive definite, the equilibrium point of (15) is locally asymptotically stable.

Proof. With (16) and (17), we choose Lyapunov functional

$$
\begin{aligned}
V(x)= & x^{\mathrm{T}} P x+\int_{t-R_{1}^{*}}^{t} x^{\mathrm{T}}(s) H x(s) \mathrm{d} s \\
& +\int_{t-R_{2}^{*}}^{t} x^{\mathrm{T}}(s) H x(s) \mathrm{d} s,
\end{aligned}
$$

then

$$
\begin{aligned}
\dot{V}= & x^{\mathrm{T}}(t)\left(A^{\mathrm{T}} P+P A\right) x(t)+2 x^{\mathrm{T}}\left(t-R_{1}^{*}\right) B_{1}^{\mathrm{T}} P x(t) \\
& +2 x^{\mathrm{T}}\left(t-R_{2}^{*}\right) B_{2}^{\mathrm{T}} P x(t)+2 x^{\mathrm{T}}(t) H x(t) \\
& -x^{\mathrm{T}}\left(t-R_{1}^{*}\right) H x\left(t-R_{1}^{*}\right)-x^{\mathrm{T}}\left(t-R_{2}^{*}\right) H x\left(t-R_{2}^{*}\right) \\
= & -x^{\mathrm{T}}(t)(Q-2 H) x(t)+2 x^{\mathrm{T}}\left(t-R_{1}^{*}\right) B_{1}^{\mathrm{T}} P x(t) \\
& +2 x^{\mathrm{T}}\left(t-R_{2}^{*}\right) B_{2}^{\mathrm{T}} P x(t)-x^{\mathrm{T}}\left(t-R_{1}^{*}\right) H x\left(t-R_{1}^{*}\right) \\
& -x^{\mathrm{T}}\left(t-R_{2}^{*}\right) H x\left(t-R_{2}^{*}\right) \\
= & -\left(x^{\mathrm{T}}(t), x^{\mathrm{T}}\left(t-R_{1}^{*}\right), x^{\mathrm{T}}\left(t-R_{2}^{*}\right)\right) \\
& \cdot\left[\begin{array}{ccc}
Q-2 H & -P B_{1} & -P B_{2} \\
-B_{1}^{\mathrm{T}} P & H & 0 \\
-B_{2}^{\mathrm{T}} P & 0 & H
\end{array}\right] \cdot\left[\begin{array}{c}
x(t) \\
x\left(t-R_{1}^{*}\right) \\
x\left(t-R_{2}^{*}\right)
\end{array}\right] .
\end{aligned}
$$

Denote $D=\left[\begin{array}{ccc}Q-2 H & -P B_{1} & -P B_{2} \\ -B_{1}^{\mathrm{T}} P & H & 0 \\ -B_{2}^{\mathrm{T}} P & 0 & H\end{array}\right]$. Thus, system (15) is locally asymptotically stable if $D$ is positive definite.

The two theorems provide sufficient conditions of local asymptotic stability for the AIMD/RED system with heterogeneous delays. It is worth pointing out that sufficient conditions derived in Theorem 4 to Theorem 7 are all given in terms of linear matrix inequalities (LMIs). These conditions can be easily assessed by applying the LMI Control Toolbox with Matlab, which makes our results of good practical value. We give a numerical example for Theorem 7: let $N_{1}=N_{2}=10, K_{p}=0.0001, C=12,000$ (packets/ s). Choose $\left(\alpha_{1}, \beta_{1}\right)=(1,0.5)$ with $T_{p 1}=0.01(\mathrm{~s})$, and $\left(\alpha_{2}, \beta_{2}\right)=(0.2,0.875)$ with $T_{p 2}=0.008(\mathrm{~s})$, respectively. Solving the LMI in Theorem 7 with Matlab
Control Toolbox, one feasible solution we obtain is as follows:

$$
Q=\left[\begin{array}{ccc}
107.8925 & 66.0119 & 49.7801 \\
66.0119 & 62.8475 & 38.7408 \\
49.7801 & 38.7408 & 52.1792
\end{array}\right]
$$

and $H=\frac{1}{4} Q$. Note that $Q$ and $H$ are positive definite. We obtain matrix

$P=\left[\begin{array}{ccc}13.8052 & 6.9367 & -0.3094 \\ 6.9367 & 11.6831 & -0.1195 \\ -0.3094 & -0.1195 & 0.1443\end{array}\right]$

with Matlab, and the eigenvalues of the matrix $D=\left[\begin{array}{ccc}Q-2 H & -P B_{1} & -P B_{2} \\ -B_{1}^{\mathrm{T}} P & H & 0 \\ -B_{2}^{\mathrm{T}} P & 0 & H\end{array}\right]$

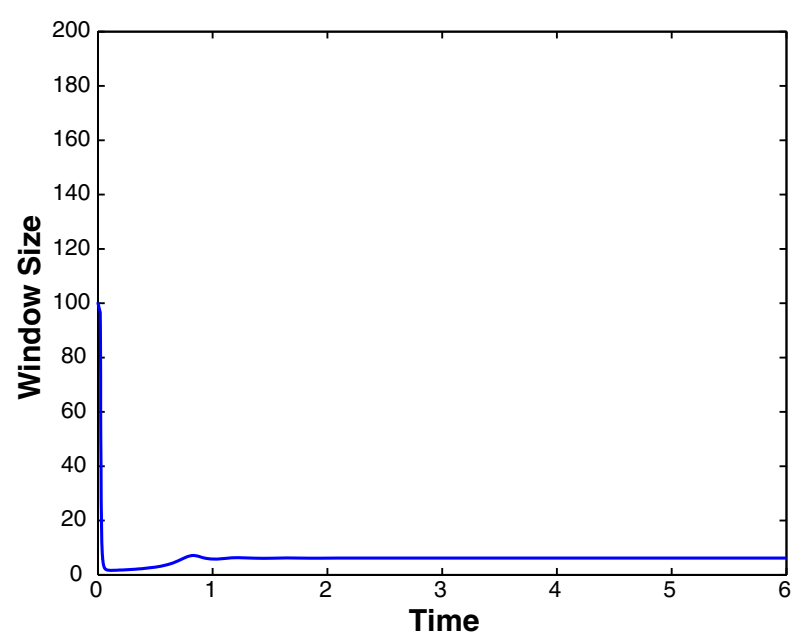

(a) Window trace

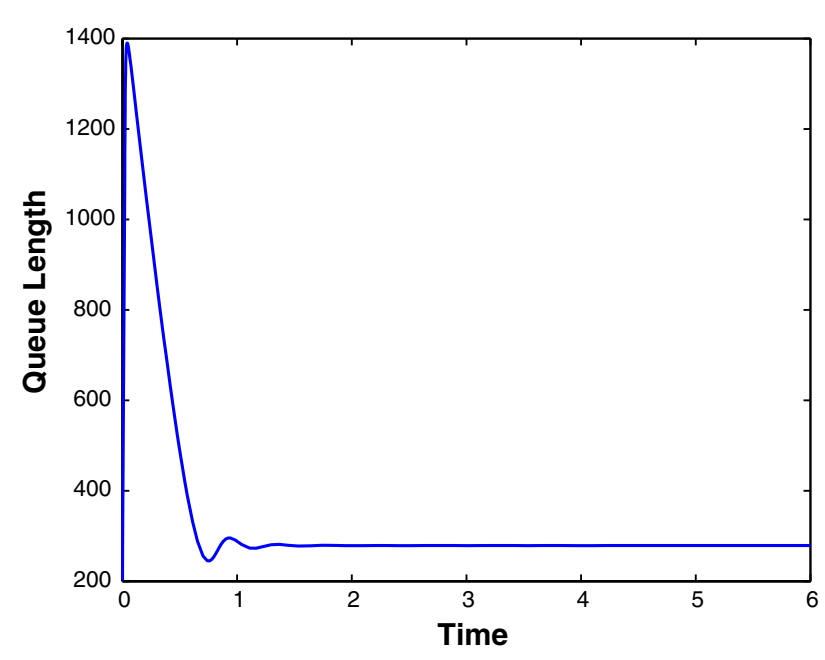

(b) Queue length

Fig. 5. TCP flows. 
are all positive: $2.4997,3.4597,3.8422,5.5610$, $7.9974,13.6734,46.2107,46.2592,93.4159$; therefore, $D$ is positive definite. Thus, the condition of Theorem 7 holds and the system is locally asymptotically stable. Simulation results using the same parameters will be give in Section 6 .

While choosing parameters in the numerical example, we have also found that link capacity $C$ and feedback delays cannot be too large, so that the matrix $D$ can be positive definite. This observation is also consistent with [3], which suggested that TCP/RED will become unstable when delay increases, or more strikingly, when link capacity increases.

Similarly, we can obtain the local stability of the AIMD/RED system when it is shared by more than two classes of heterogeneous flows as well. The proof is omitted here.

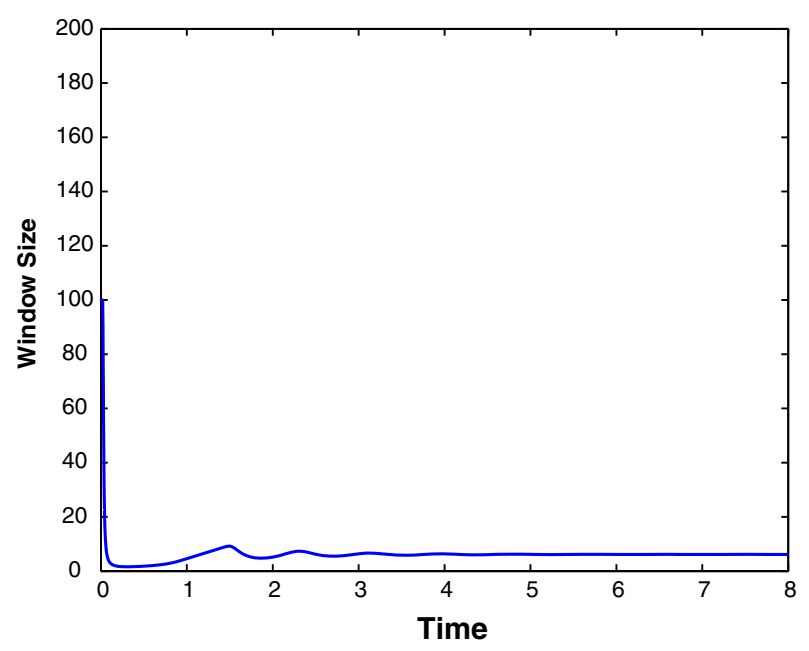

(a) Window trace

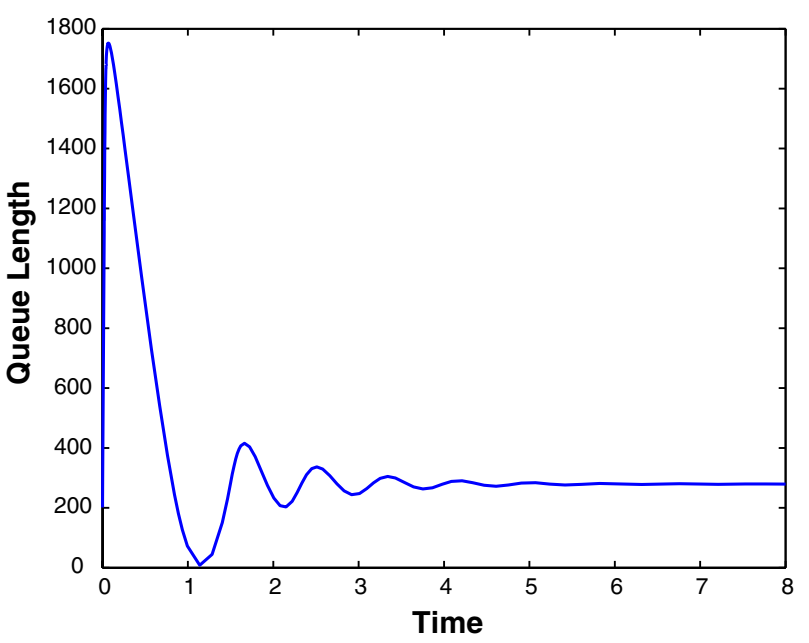

(b) Queue length

Fig. 6. $\operatorname{AIMD}(0.2,0.875)$ flows.

\subsection{TCP-friendliness}

According to the equilibrium point of the system, $W_{\mathrm{I}}^{*} / W_{\mathrm{II}}^{*}=G$ is a function of the AIMD parameter pairs, and it is independent of the delays. In other words, for two AIMD flows, as long as their AIMD parameters satisfy the condition that $G=1$, their average window sizes are the same and their flow throughputs inversely proportional to their rtts. To be TCP-friendly, the necessary and sufficient condition is still $\alpha=3(1-\beta) /(1+\beta)$, the same as the condition (10) derived in the delay-free systems in Section 4.3.

\section{Performance evaluation}

Matlab is used to obtain the system evolution trajectory of the fluid model in order to verify the

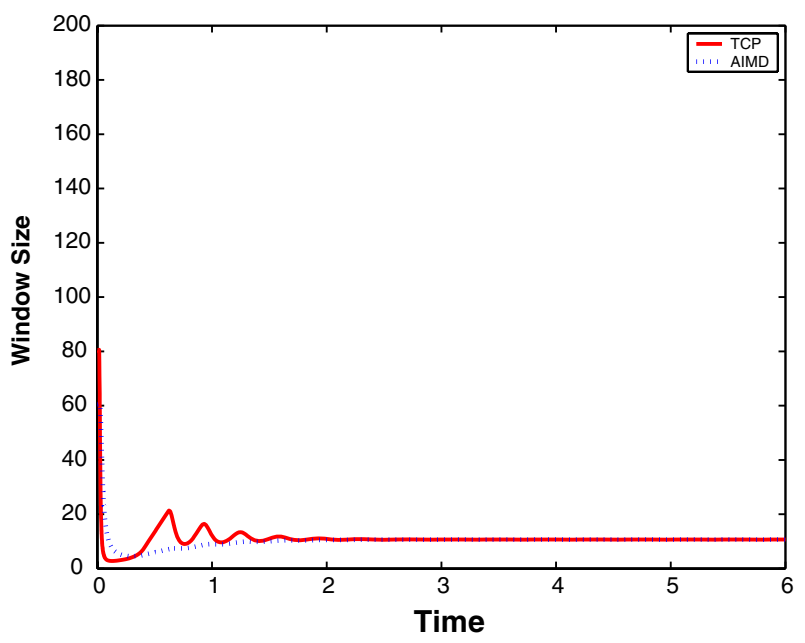

(a) Window trace

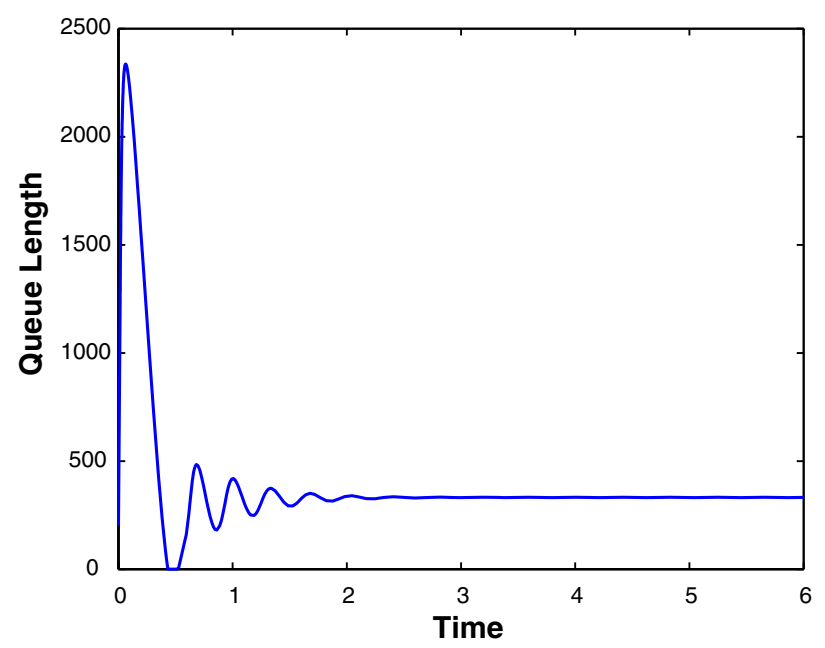

(b) Queue length

Fig. 7. TCP vs. $\operatorname{AIMD}(0.2,0.875)$ flows. 
asymptotic stability proved in Section 5. Network simulator, NS-2, is used to evaluate the performance of the AIMD/RED systems.

\subsection{Numeric results}

The traces of window size and queue length of 10 TCP flows and $10 \operatorname{AIMD}(0.2,0.875)$ flows in a RED-enabled link with feedback delays are given in Figs. 5 and 6, respectively. The parameters used are the same as those in the numerical example of Theorem 5, i.e., $C=3000$ packet/s, $K_{p}=0.0005$, $r t t=0.02 \mathrm{~s}$, and $\min _{\mathrm{th}}=200$ packets. For heterogeneous-flow case, let 10 TCP flows and 10 $\operatorname{AIMD}(0.2,0.875)$ flows share the bottleneck with $C=12,000$ packet/s, $K_{p}=0.0001$, and rtts of the TCP and AIMD flow are $0.01 \mathrm{~s}$ and $0.008 \mathrm{~s}$, respectively. These parameters are the same as those in the numerical example of Theorem 7. To show the local asymptotic stability of the system, we choose the value of the initial condition close to the equilibrium

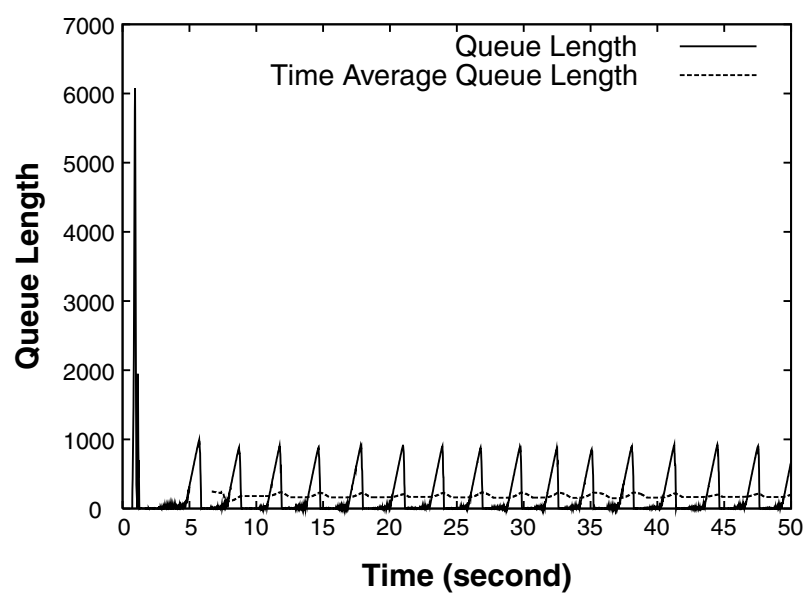

(a) Queue length

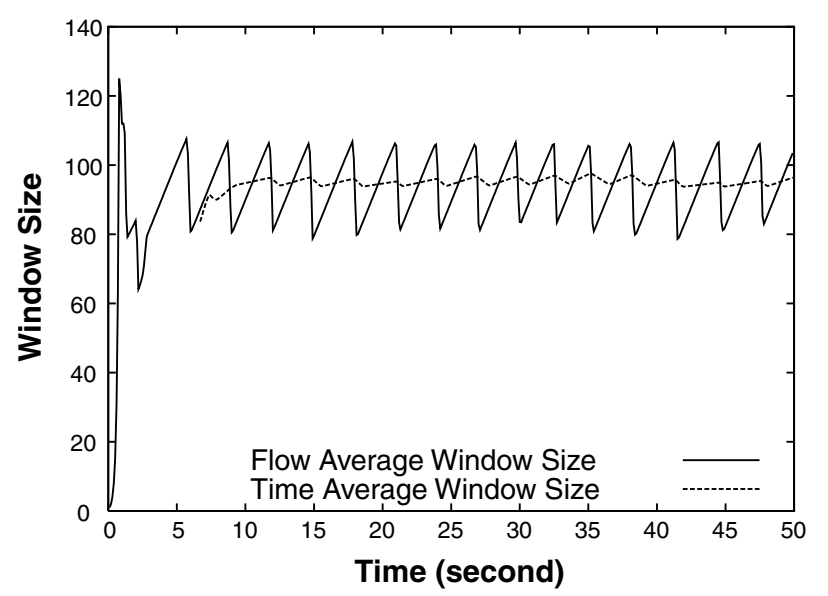

(b) Window trace

Fig. 8. TCP, $K_{p}=0.0001, R=100 \mathrm{~ms}$. point. As shown in the figures, all systems are asymptotically stable, and the numerical results validate the theorems proved in the paper. Since the parameter pair $(0.2,0.875)$ satisfies the TCP-friendly condition derived, the average window sizes of the competing TCP and AIMD $(0.2,0.875)$ flows should be the same, which is verified by the numerical results shown in Fig. 7.

\subsection{Simulation results}

We use network simulator (NS-2) to further study the performance of the AIMD/RED system with realistic protocols and network topologies. Both single bottleneck and multiple bottleneck topologies are used in the simulations. The following parameters are used unless otherwise explicitly stated. The routers adjacent to the bottleneck link are RED-capable: all packets can be queued when the average queue length is less than 200 packets,

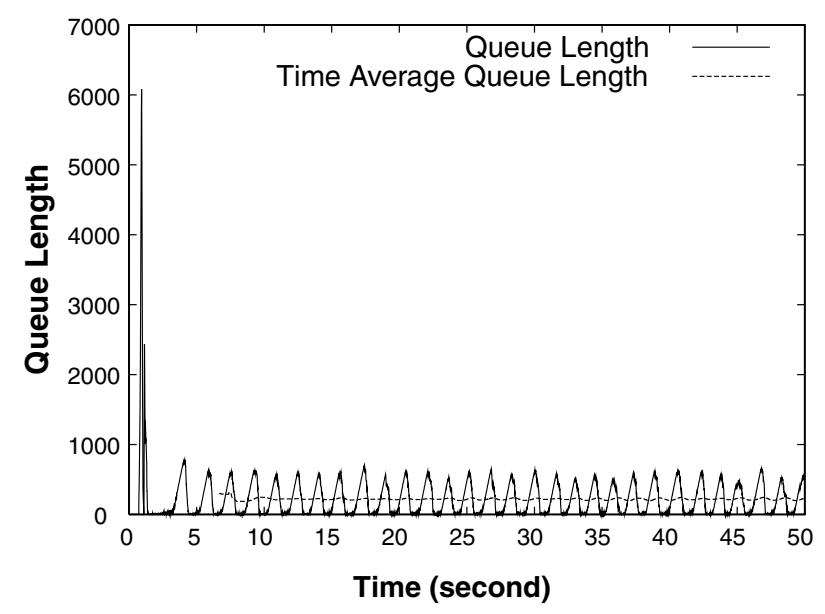

(a) Queue length

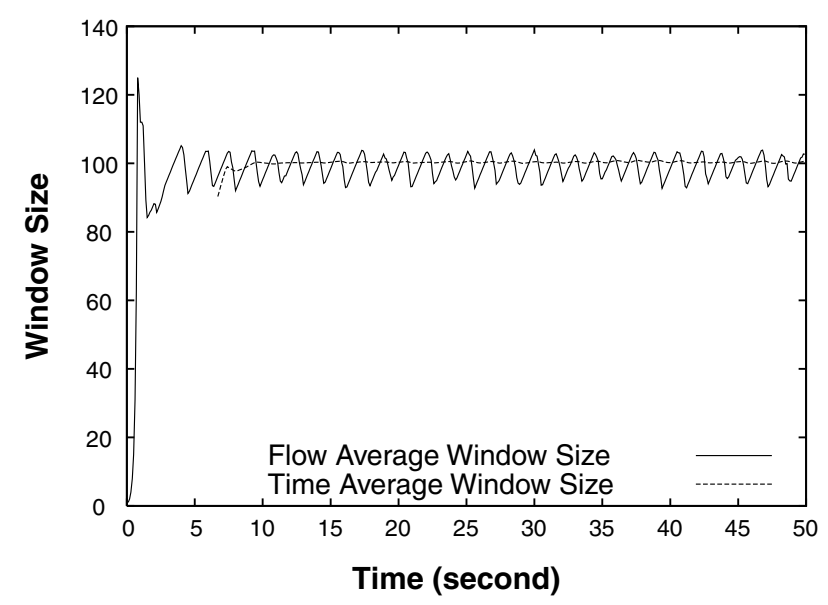

(b) Window trace

Fig. 9. TCP, $K_{p}=0.00002, R=100 \mathrm{~ms}$. 
and the packets will be discarded with probability $K_{p}$ times the current average queue length minus 200. The packet size of all flows is 1250 bytes. The bottleneck link capacity is $1 \mathrm{Gbps}$, equivalent to 100,000 packet/s.

We first let 100 TCP flows and 100 $\operatorname{AIMD}(0.2,0.875)$ flows with homogeneous delays share a single bottleneck, respectively. Their window traces and instantaneous queue lengths are given in Figs. 8-11, with different values of $r t t$ and $K_{p}$. All figures show that the flow window sizes and queue lengths are periodically oscillating in steady state, and their time averages over a round are converging to certain values, i.e., their time averages are asymptotically stable.

As shown in Figs. 8 and 9, a small value of $K_{p}$ can reduce the oscillation amplitude in the steady state, and thus improve the link utilization and reduce delay jitter in the steady state, at the cost of taking longer for the system to reach the steady state. The network utilization in transient states is

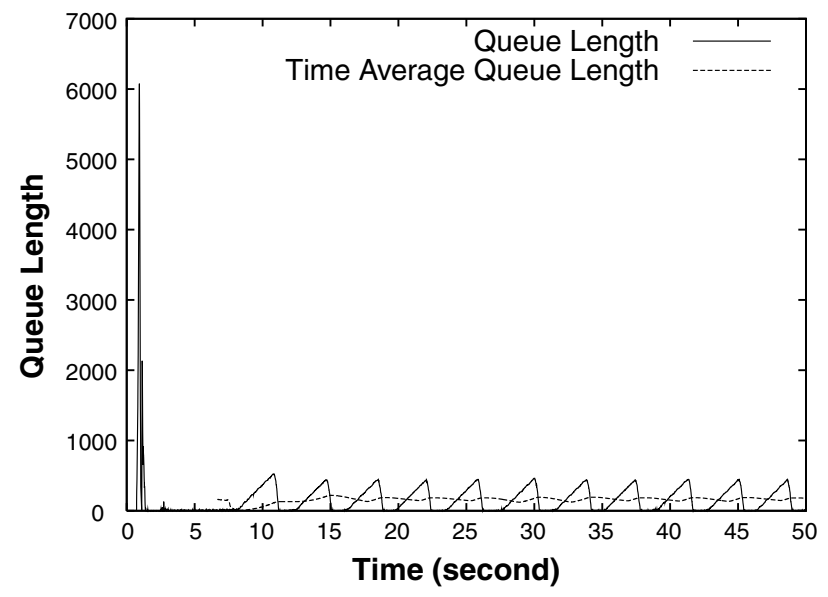

(a) Queue length

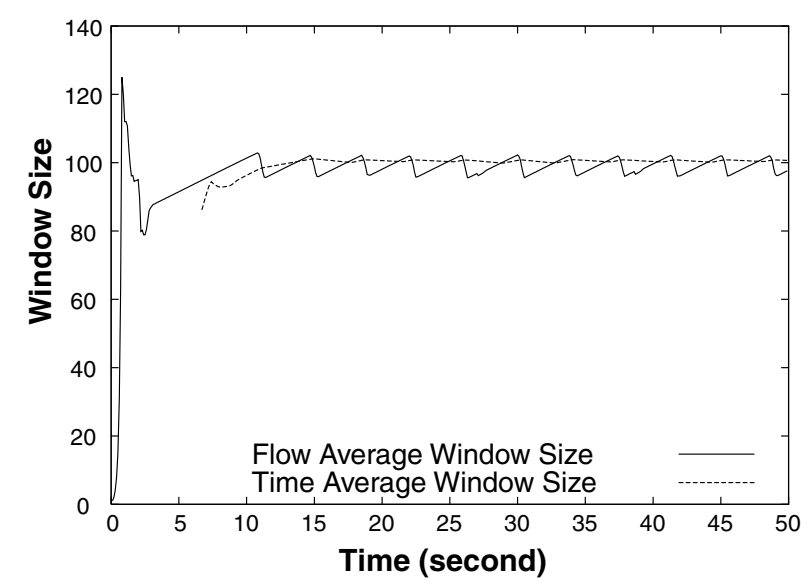

(b) Window trace

Fig. 10. $\operatorname{AIMD}(0.2,0.875), K_{p}=0.0001, R=100 \mathrm{~ms}$.

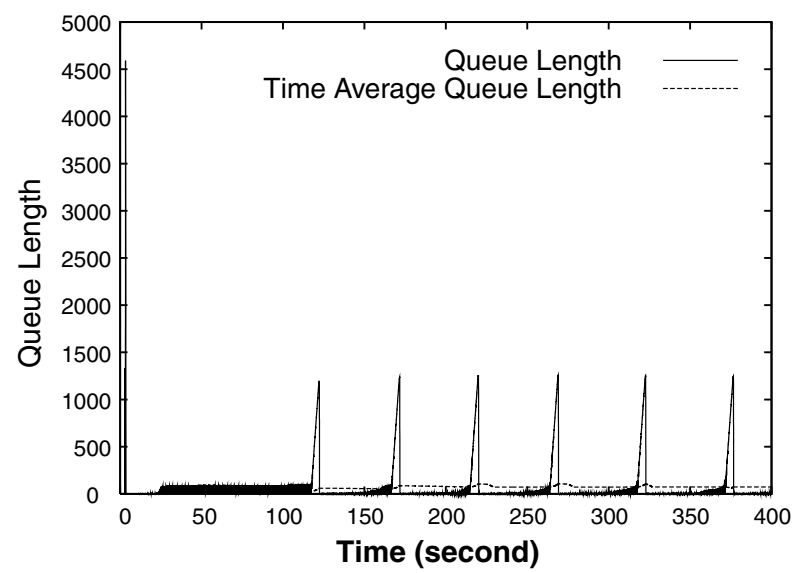

(a) Queue length

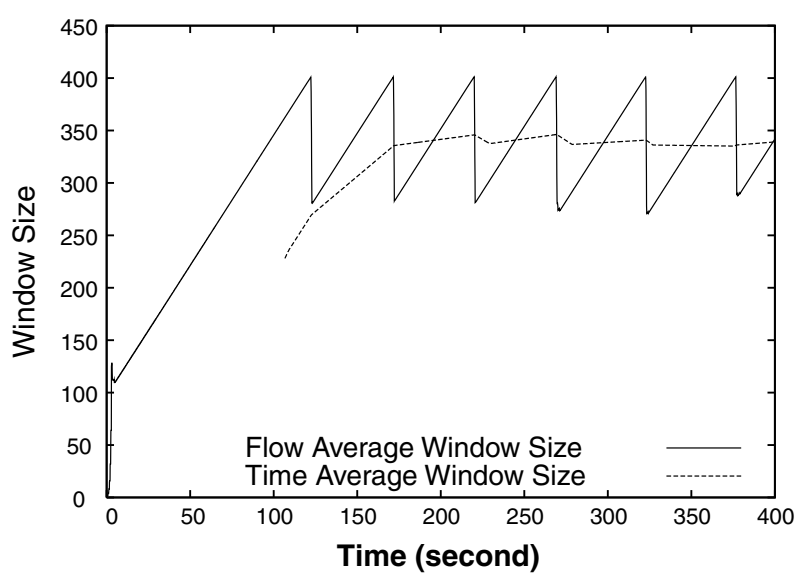

(b) Window trace

Fig. 11. TCP, $K_{p}=0.0001, R=400 \mathrm{~ms}$.

low, so a slow convergence speed is not desired. Comparing Figs. 8 and 10, it is noticed that the system with AIMD $(0.2,0.875)$ flows has smaller oscillation amplitude in the steady state because the AIMD flows have a smaller value of $\alpha$ and a larger value of $\beta$ than that of TCP flows. Another observation from Figs. 8 and 11 is that the larger the rtt, the slower the system converges to the steady state.

To study the system performance with heterogeneous flows, let $24 \operatorname{AIMD}(0.2,0.875)$ flows compete with 100 TCP flows, and their rtts are randomly chosen between $0.09 \mathrm{~s}$ and $0.1 \mathrm{~s}$. The traces of their average window size and queue length are given in Fig. 12. It is shown that, when heterogeneous TCP and $\operatorname{AIMD}(0.2,0.875)$ flows share the network, the network converges to the steady state quickly and the queue oscillation in the steady state is small. In other words, when heterogeneous traffic shares the network, the system performance is even better than that with only TCP flows (high oscillation amplitude in the steady state) or homogeneous AIMD $(0.2,0.875)$ flows (slow convergence speed). 


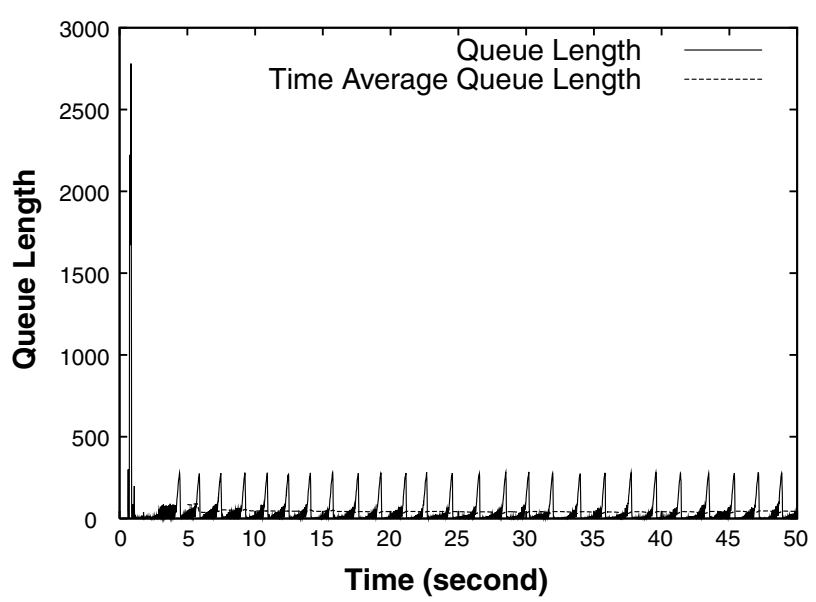

(a) Queue length

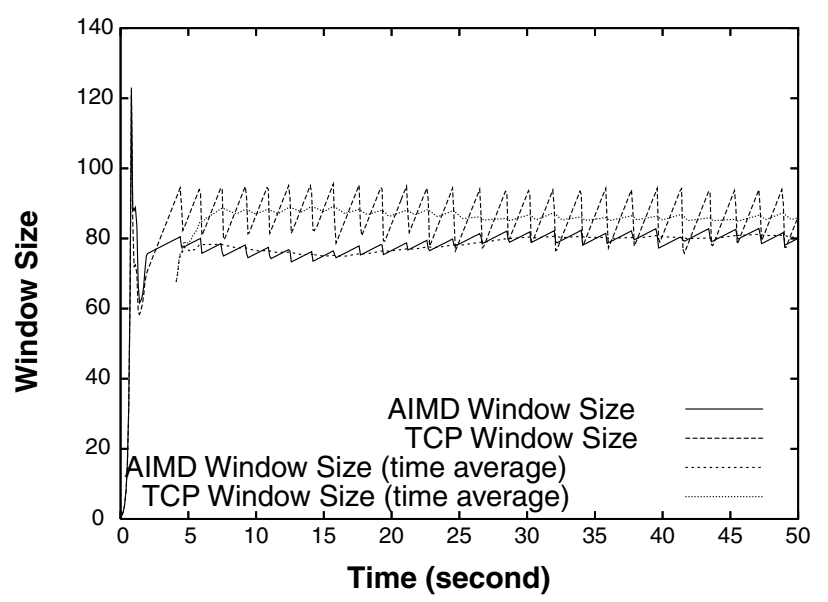

(b) Window trace

Fig. 12. TCP and $\operatorname{AIMD}(0.2,0.875)$ flows.

Another observation from Fig. 12 is that the average window sizes of the TCP flows and the AIMD $(0.2,0.875)$ flows are close to each other, therefore validating the TCP-friendly condition derived in Section 4.

A realistic network will accommodate flows with heterogeneous round-trip delays, and some flows may undergo multiple bottlenecks. The topology used for a multiple-bottleneck scenario is shown in Fig. 13; 100 group I flows compete with 50 group II TCP flows in link $r_{0} r_{1}$ and with 50 group III TCP flows in link $r_{1} r_{2}$. The round-trip times of the flows are randomly chosen from $50 \mathrm{~ms}$ to $400 \mathrm{~ms}$. There are $50 \mathrm{TCP}$ flows and $50 \operatorname{AIMD}(0.2,0.875)$ flows in group I. The trace of queue length at $r_{0}$ is shown in Fig. 14. Although the instantaneous queue length oscillates over time, the time average does not change significantly. The stability conditions for multiple-bottleneck AIMD/RED systems are under investigation.

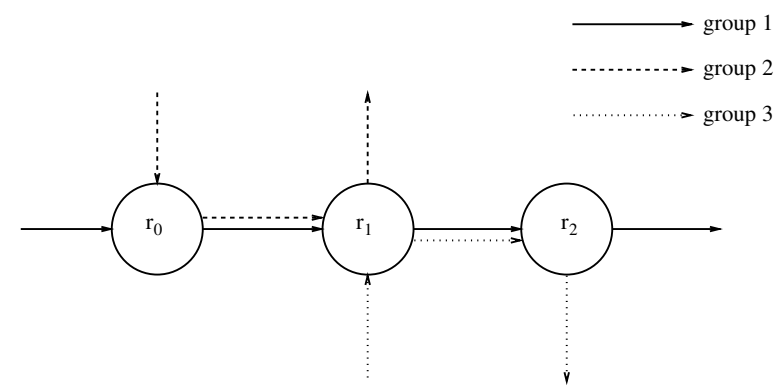

Fig. 13. Queue length, multiple-bottleneck topology.

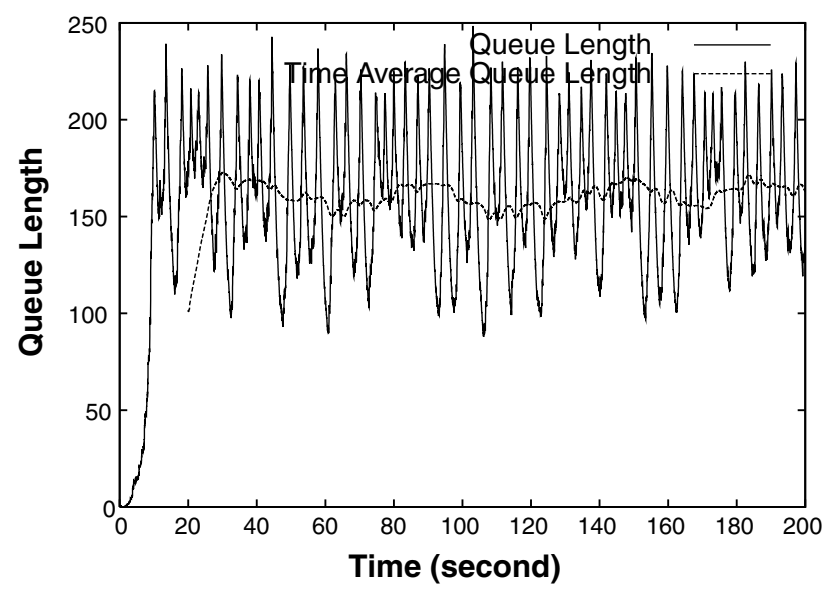

Fig. 14. Multiple-bottleneck, heterogeneous round-trip delays.

\section{Conclusions and future work issues}

In this paper, we have studied the stability of AIMD/RED systems with and without the consideration of feedback delays. Delay-free systems have been proved asymptotically stable. Sufficient conditions have been obtained for the local asymptotic stability of both homogeneous-flow and heterogeneous-flow systems with feedback delays, which provide insight and guidelines for the design of a stable system. TCP-friendliness issue has also been discussed for multiple flows with different AIMD parameters and different rtts. Numerical results have been given to validate the analytical results, and extensive simulations with NS-2 have been conducted to study the system performance with realistic protocols and network topologies. The study will be useful to re-design and re-engineer TCP congestion control for supporting heterogeneous multimedia application in more diversified Internet in the future.

There are many interesting open issues require further research. First, for RED queues, the packet drop probability depends on the queue length only. With the model presented in the paper, the average 
queue length in the steady state can be derived, which can be used to give a rough estimation of the packet loss rate. However, the packet loss rate depends on the queue length distribution, which is unknown from the model. Second, the robustness of the system with disturbance from short-lived TCP connections and UDP connections is an important open issue. Third, a single-bottleneck topology is used in this paper. In a follow-up work, we will extend the stability analysis to systems with multiple bottlenecks. Finally, since multicast applications may use a large portion of Internet bandwidth in the future, how to design and analyze flow/congestion control mechanisms for multicast applications is a very challenging issue beckon for more research.

\section{Acknowledgement}

This work has been supported in part by a research grant from the Natural Science and Engineering Council of Canada.

\section{References}

[1] D.M. Chiu, R. Jain, Analysis of the increase and decrease algorithms for congestion avoidance in computer networks, Computer Networks and ISDN Systems 17 (1) (1989) 1-14.

[2] R. Johari, D.K.H. Tan, End-to-end congestion control for the internet: delays and stability, IEEE/ACM Transactions on Networking 9 (6) (2001) 818-832.

[3] S. Low, F. Paganini, J. Wang, S. Adlakha, J.C. Doyle, Dynamics of TCP/RED and a scalable control, IEEE Infocom'02 1 (June) (2002) 239-248.

[4] S. Floyd, M. Handley, J. Padhye, A comparison of equationbased and AIMD congestion control, May 2000. <http:// www.aciri.org/tfrc/tcp-friendly.TR.ps>.

[5] Y.R. Yang, S.S. Lam, General AIMD congestion control. Technical Report TR-2000-09, University of Texas, May 2000. A shorter version appeared in Proceedings of ICNP'00, Osaka, Japan, November 2000.

[6] L. Cai, X. Shen, J. Pan, J.W. Mark, Performance analysis of TCP-friendly AIMD algorithms for multimedia applications, IEEE Transactions on Multimedia 7 (2) (2005) 339-355. April.

[7] S. Floyd, S. McCanne, Network simulator, LBNL Public Domain Software. Available via ftp from ftp.ee.lbl.gov. NS2. $<$ http://www.isi.edu/nsnam/ns/>.

[8] V. Firoiu, M. Borden, A study of active queue management for congestion control, in: Proceedings of Infocom, vol. 3, March 2000, pp. 1435-1444.

[9] C. Wang, J. Liu, B. Li, K. Sohraby, Y.T. Hou, LRED: A robust and responsive aqm algorithm using packet loss ratio measurement, IEEE Transactions on Parallel and Distributed Systems 18 (1) (2007) 29-43.

[10] S. Floyd, V. Jacobson, Random early detection gateways for congestion avoidance, IEEE/ACM Transactions on Networking 1 (August) (1993) 397-413.
[11] D. Lin, R. Morris, Dynamics of random early detection, Proceedings of ACM/SIGCOMM (1997) 127-137.

[12] F.P. Kelly, A.K. Maulloo, D.K.H. Tan, Rate control for communication networks: shadow prices, proportional fairness and stability, Journal of the Operational Research Society 49 (3) (1998) 237-252.

[13] S. Deb, R. Srikant, Global stability of congestion controllers for the internet, IEEE Transactions on Automatic Control 48 (6) (2003) 1055-1060.

[14] L. Ying, G.E. Dullerud, R. Srikant, Global stability of internet congestion controllers with heterogeneous delays, American Control Conference 4 (June) (2004) 2948-2953.

[15] Y. Zhang, S. Kang, D. Loguinov, Delayed stability and performance of distributed congestion control, in: Proceedings of the 2004 Conference on Applications, Technologies, Architectures, and Protocols for Computer Communications, 2004, pp. 307-318.

[16] Z. Wang, F. Paganini, Global stability with time-delay in network congestion control, in: Proceedings of IEEE Conference on Decision and Control, LasVegas, NV, vol. 4, December, 2002, pp. 3632-3637.

[17] S. Low, A duality model of TCP and queue management algorithms, IEEE Transactions on Networking 11 (4) (2003) 525-536.

[18] V. Misra, W.B. Gong, D. Towsley, Fluid-based analysis of a network of AQM routers supporting TCP flows, Proceedings of ACM/SIGCOMM (2000) 151-160.

[19] C.V. Hollot, V. Misra, D. Towsley, W.B. Gong, Analysis and design of controllers for AQM routers supporting TCP flows, IEEE Transactions on Automatic Control 47 (6) (2002) 945-959.

[20] C.V. Hollot, V. Misra, D. Towsley, W.B. Gong, On designing improved controllers for AQM routers supporting TCP flows, in: Proceedings of IEEE INFOCOM, April 2001, pp. 1726-1734, Anchorage.

[21] C.V. Hollot, V. Misra, D. Towsley, W.B. Gong, A control theoretic analysis of RED, in: Proceedings of IEEE INFOCOM, April 2001, pp. 1510-1519, Anchorage.

[22] C.V. Hollot, Y. Chait, Non-linear stability analysis of a class of TCP/AQM networks, in: Proceedings of IEEE Conference on Decision and Control, vol. 3, December 2001, Orlando, Florida USA, pp. 2309-2314.

[23] Y. Chait, C.V. Hollot, V. Misra, S. Oldak, D. Towsley, W.B. Gong, Fixed and adaptive model-based controllers for active queue management, Proceedings of ACC 4 (2001) 29812986.

[24] D.G. Luenberger, Introduction to dynamic systems: theory, models, and application, May 1979. ISBN 0-471-02594-1.

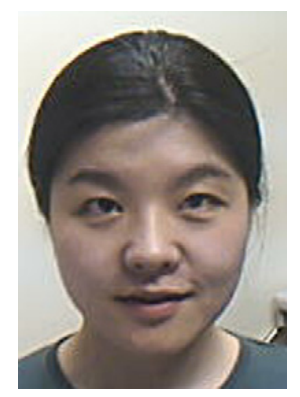

Lijun Wang received her B.Sc. degree in Computer Science and M.Sc. degree in Mathematics from Shandong University, China, in 2000 and 2003, respectively. She is currently pursuing the Ph.D. degree in the department of Applied Mathematics at the University of Waterloo, Waterloo, ON, Canada. Her research interests is mainly on the congestion control problem in the Internet, and stability analysis of nonlinear systems and hybrid dynamic systems. 


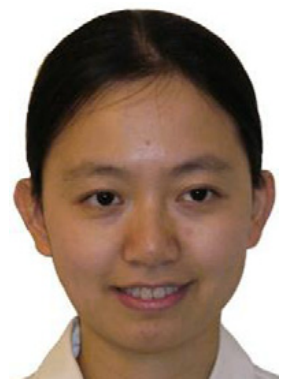

Lin Cai received the M.A.Sc. and Ph.D. degrees (with Outstanding Achievement in Graduate Studies Award) in electrical and computer engineering from the University of Waterloo, Waterloo, Canada, in 2002 and 2005, respectively. Since July 2005, she has been an Assistant Professor in the Department of Electrical and Computer Engineering at the University of Victoria, British Columbia, Canada. Her research interests span several areas in wireless communications and networking, with a focus on network protocol and architecture design supporting emerging multimedia traffic over wireless, mobile, ad hoc, and sensor networks. She serves as the Associate Editor for IEEE Transactions on Vehicular Technology (2007- ), EURASIP Journal on Wireless Communications and Networking (2006- ), and International Journal of Sensor Networks (2006- ).

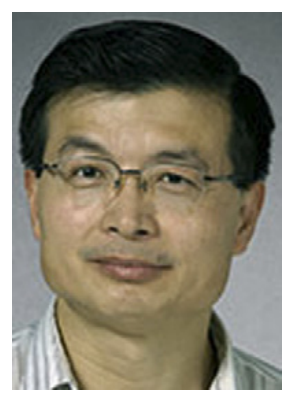

Xinzhi Liu received the B.Sc. degree in mathematics from Shandong Normal University, Jinan, China, in 1982, and the M.Sc. and Ph.D. degrees, all in applied mathematics, from University of Texas, Arlington, in 1987 and 1988, respectively. He was a Post-Doctoral Fellow at the University of Alberta, Edmonton, AB, Canada, from 1988 to 1990. He joined the Department of Applied Mathematics, University of Waterloo, Waterloo, ON, Canada, in 1990, where he became an Associate Professor in 1994, and a Professor in 1997. His research areas include systems analysis, stability theory, hybrid dynamical systems, impulsive control, chaos synchronization, nonlinear oscillations, artificial neural networks, and communication security. He is the author or coauthor of over 150 research articles and two research monographs and five other books. He is the Chief Editor of the journal, DCDIS Series A: Mathematical Analysis, and the Chief Editor of the Journal, DCDIS Series B: Applications and Algorithms, and Associate Editor of four other journals. He served as General Chair for several international scientific conferences.

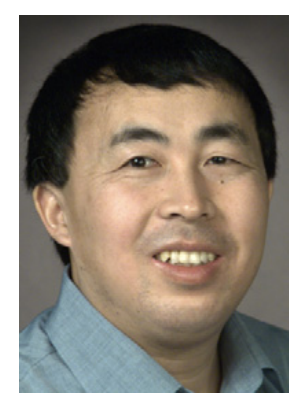

Xuemin (Sherman) Shen received the B.Sc. (1982) degree from Dalian Maritime University (China) and the M.Sc. (1987) and Ph.D. degrees (1990) from Rutgers University, New Jersey (USA), all in electrical engineering. He is a Professor and the Associate Chair for Graduate Studies, Department of Electrical and Computer Engineering, University of Waterloo, Canada. His research focuses on mobility and resource management in interconnected wireless/wired networks, UWB wireless communications systems, wireless security, and ad hoc and sensor networks. He is a co-author of three books, and has published more than 300 papers and book chapters in wireless communications and networks, control and filtering. Dr. Shen serves as the Technical Program Committee Chair for IEEE Globecom'07, General Co-Chair for Chinacom'07 and QShine'06, the Founding Chair for IEEE Communications Society Technical Committee on P2P Communications and Networking. He also serves as a Founding Area Editor for IEEE Transactions on Wireless Communications; Editor-in-Chief for Peer-to-Peer Networking and Application; Associate Editor for IEEE Transactions on Vehicular Technology; KICS/IEEE Journal of Communications and Networks, Computer Networks; ACM/Wireless Networks; and Wireless Communications and Mobile Computing (Wiley), etc. He has also served as Guest Editor for IEEE JSAC, IEEE Wireless Communications, and IEEE Communications Magazine. He received the Excellent Graduate Supervision Award in 2006, and the Outstanding Performance Award in 2004 from the University of Waterloo, the Premier's Research Excellence Award (PREA) in 2003 from the Province of Ontario, Canada, and the Distinguished Performance Award in 2002 from the Faculty of Engineering, University of Waterloo. Dr. Shen is a registered Professional Engineer of Ontario, Canada. 